\title{
Loss of Function of P2X7 Receptor Scavenger Activity in Aging Mice
}

\section{A Novel Model for Investigating the Early Pathogenesis of Age-Related Macular Degeneration}

\author{
Kirstan A. Vessey, ${ }^{*}$ Ben J. Gu, ${ }^{\dagger}$ Andrew I. Jobling, ${ }^{*}$ Joanna A. Phipps, ${ }^{*}$ Ursula Greferath, ${ }^{*}$ Mai X. Tran, ${ }^{*}$ Michael A. Dixon, ${ }^{*}$
} Paul N. Baird, ${ }^{\ddagger \S}$ Robyn H. Guymer, ${ }^{\ddagger}$ James S. Wiley, ${ }^{\dagger}$ and Erica L. Fletcher*

From the Department of Anatomy and Neuroscience* and the Division of Ophthalmology, ${ }^{\S}$ Department of Surgery, The University of Melbourne, Melbourne; the Florey Institute of Neuroscience, ${ }^{\dagger}$ Melbourne; and the Centre for Eye Research Australia, ${ }^{\ddagger}$ Royal Victorian Eye and Ear Hospital, Melbourne, Victoria, Australia

\author{
Accepted for publication \\ April 13, 2017. \\ Address correspondence to \\ Erica L. Fletcher, Ph.D., \\ Department of Anatomy and \\ Neuroscience, The University \\ of Melbourne, Level 2, Medical \\ Bldg, Grattan St, Parkville, VIC \\ 3010, Australia. E-mail: elf@ \\ unimelb.edu.au.
}

\begin{abstract}
Age-related macular degeneration (AMD) is a leading cause of irreversible, severe vision loss in Western countries. Recently, we identified a novel pathway involving $\mathrm{P} 2 \mathrm{X} 7$ receptor scavenger function expressed on ocular immune cells as a risk factor for advanced AMD. In this study, we investigate the effect of loss of P2X7 receptor function on retinal structure and function during aging. P2X7-null and wild-type C57bl6J mice were investigated at 4, 12, and 18 months of age for macrophage phagocytosis activity, ocular histological changes, and retinal function. Phagocytosis activity of blood-borne macrophages decreased with age at 18 months in the wild-type mouse. Lack of P2X7 receptor function reduced phagocytosis at all ages compared to wild-type mice. At 12 months of age, P2X7-null mice had thickening of Bruchs membrane and retinal pigment epithelium dysfunction. By 18 months of age, P2X7-null mice displayed phenotypic characteristics consistent with early AMD, including Bruchs membrane thickening, retinal pigment epithelium cell loss, retinal functional deficits, and signs of subretinal inflammation. Our present study shows that loss of function of the P2X7 receptor in mice induces retinal changes representing characteristics of early AMD, providing a valuable model for investigating the role of scavenger receptor function and the immune system in the development of this age-related disease. (Am J Pathol 2017, 187: 1670-1685; http://dx.doi.org/10.1016/j.ajpath.2017.04.016)
\end{abstract}

Age-related macular degeneration (AMD) is one of the leading causes of irreversible vision loss in the Western world. ${ }^{1,2}$ The disease is defined by the initial appearance of extracellular deposits, called drusen, between the retinal pigment epithelium (RPE) basal lamina and the internal collagenous layer of Bruchs membrane that are composed of glycoproteins, lipids, and immunogenic factors. ${ }^{3,4}$ Further pathology follows, resulting in death of cells that are critical for visual function: the RPE and the light-sensitive photoreceptors. There are two clinically distinct forms of endstage disease, where vision is threatened: neovascular and atrophic AMD. ${ }^{1,2}$ Atrophic AMD is a slowly developing condition whereby the RPE and the photoreceptors die, particularly in the specialized region of the central retina called the macula, resulting in vision loss. In contrast, neovascular AMD occurs when blood vessels grow into the retina, leak, and cause widespread destruction of the outer

\footnotetext{
Supported by National Health and Medical Research Council (NHMRC) P2X7-grants APP1061419 (E.L.F., K.A.V., B.J.G., and R.H.G.) and APP1120095 (R.H.G, E.L.F. B.J.G, and K.A.V); the Macular Disease Foundation of Australia, 2013 (E.L.F., K.A.V., B.J.G., R.H.G., and P.N.B.); NHMRC Senior Research Fellowship number 1028444 (P.N.B.); and NHMRC Principal Research Fellowship number 1103013 (R.H.G.). The Center for Eye Research Australia receives operational infrastructure support from the Victorian government.
}

Disclosures: None declared. 
retinal layers, leading to significant neuronal cell death and rapid central vision loss. Regardless of the end stage of the disease, there are common characteristic pathologies associated with the initial stages of early AMD (namely, the deposition of drusen between the RPE and Bruchs membrane and thickening of Bruchs membrane, a five-layered semipermeable structure delineated by and including the choriocapillaris and RPE basement membranes). ${ }^{5-11}$ With age, but more so in AMD, Bruchs membrane thickens with an accumulation of lipid-rich debris such that the ability to pass nutrients and oxygen through it is dramatically reduced. ${ }^{3,4,12}$ The causes of the drusen deposition and Bruchs membrane thickening are not fully understood but are thought to be integral to changes in the RPE and the pathogenesis of AMD.

The RPE provides an important support role for the neuronal retina, delivering nutrients from the choroidal blood supply to the photoreceptors, removing metabolic end products from photoreceptors, producing melanin granules to absorb stray light, and recycling molecules important for maintenance of the visual cycle. ${ }^{13}$ It also modulates the extracellular matrix that forms Bruchs membrane. In particular, the RPE plays an integral role in the daily renewal of the photoreceptors. Each day, a portion of the photoreceptor outer segments is engulfed by the RPE via phagocytosis and recycled and returned to the photoreceptors or delivered via diffusion through Bruchs membrane to the choroid. ${ }^{14}$ With age, and in particular in AMD, debris accumulates on the basal side of the RPE. One current hypothesis is that to aid the aging RPE, blood-borne macrophages in the choroid, which are thought to have the ability to migrate toward and engulf insoluble material found in drusen, ${ }^{15,16}$ might fail to do so and that failure of molecular pathways involved in this process might predispose individuals to development of AMD.

Recently, we have described a novel putative pathway implicating this aspect of the innate immune system in modulating the development of AMD. We have identified defective phagocytosis by peripheral blood macrophages as potentially contributing to the buildup of debris in Bruchs membrane, which is integral to the disease process leading to AMD. ${ }^{17}$ The P2X7 receptor expressed on macrophages/ microglia can act as a scavenger receptor, conferring phagocytic ability to immune cells expressing it because of a complement of six exposed cysteine residues. ${ }^{18}$ This scavenger activity occurs in the absence of ATP, the native ligand for this receptor. ${ }^{19,20}$ Recently, we found a genetic association of functional polymorphisms in the $P 2 R X 7$ and related $P 2 R X 4$ genes in a cohort of caucasian patients with late-stage AMD. Specifically, this work revealed a unique and rare haplotype containing the P2X4 315Cys plus P2X7 150Arg variants overrepresented in AMD compared with agematched controls. ${ }^{17}$ The macrophages in the blood of $\mathrm{P} 2 \mathrm{X} 7 / \mathrm{P} 2 \mathrm{X} 4$ heterozygote participants had reduced phagocytosis activity, compared to those without the polymorphisms. Our data in cell culture expression assays indicate that the P2X4-wild-type (WT) or mutant receptor, when transfected into HEK-293 cells, has little/no phagocytic activity, whereas the P2X7-WT receptor confers phagocytic activity to these otherwise non-phagocytic cells. Coexpression of the mutant P2X4 315Cys and P2X7 150Arg reduces the innate phagocytic activity of these cells. The deleterious effect of the P2X4 315Cys on P2X7-mediated phagocytosis is likely to be related to the cysteine residue on the P2X4 receptor, which may form a mixed disulfide bond with a cysteine residue in the extracellular domain of the $\mathrm{P} 2 \mathrm{X} 7$ receptor. This bond thereby links the two molecules and inhibits the scavenger function of P2X7-WT and, to a much greater extent, P2X7 150Arg receptor. ${ }^{17}$ These data suggest that the P2X7 receptors expressed on microglia/ macrophages normally function as scavenger receptors and that functional alterations in this process ultimately predispose individuals to AMD ${ }^{17}$ Our hypothesis is that with age, the RPE is no longer able to manage the task of removal of the constant supply of photoreceptor debris, and that macrophages are required to provide an additional mechanism to prevent progressive and damaging accumulation of debris within Bruchs membrane and drusen. When macrophage scavenger activity is reduced, there is ongoing debris accumulation and Bruchs membrane thickening, which leads to worse nutrient and oxygen support for the RPE and a vicious cycle of RPE failure and ultimate death.

In the current study, our aim was to characterize this novel mechanism in a mouse model and determine whether loss of the P2X7 receptor would result in an age-dependent accumulation of debris within Bruchs membrane, leading to RPE dysfunction and RPE cell loss. Loss of P2X7 receptor function in the mouse ( $\mathrm{P} 2 \mathrm{X} 7$ null) reduced blood-borne macrophage phagocytosis activity. At 12 months of age, P2X7-null mice displayed thickening of Bruchs membrane, RPE dysfunction, and retinal stress. By 18 months of age, the P2X7-null mouse displayed phenotypic characteristics consistent with early AMD, including Bruchs membrane thickening, RPE cell loss, retinal functional deficits, and signs of inflammation between the RPE and photoreceptors. Our data suggest that bloodborne macrophages aid the aging RPE in the clearance of debris and that failure of this aspect of the innate immune system gives impetus to a pathological pathway relentlessly driving further debris accumulation and RPE failure.

\section{Materials and Methods}

\section{Animals}

C57bl6J mice (WT mice) were obtained from the Animal Resource Center (Murdoch, WA, Australia). P2X7-null mice on a C57bl6J background were a gift from Prof. James Wiley (The Florey Institute of Neuroscience, Melbourne, Australia). ${ }^{21}$ The P2X7-null mice have an insertion in exon 13 of the P2X7 receptor gene (official name P2RX7), which deletes from Cys506 to Pro532 in the $\mathrm{C}$ terminus of the 595-amino acid protein. Before experimentation, the P2X7-null mice were rederived using Animal Resource 
Center C57bl6J mice and also then backcrossed to the Animal Resource Center C57bl6J mouse strain before homozygous P2X7-null mice were generated and compared with the Animal Resource Center C57bl6J mouse strain. All mice were bred and housed at The University of Melbourne (Melbourne, VIC, Australia) animal facility on a 12-hour light/dark cycle. Food and water were available ad libitum. All experiments adhered to the American Association of Vision Research and Ophthalmology standards for the ethical treatment of animals and the ethics committee standards of The University of Melbourne (number 1313030). Both male and female mice were used and evenly balanced for sex for each experiment. Mice were aged to 4, 12, and 18 months.

\section{Fundus Imaging}

To view the integrity of the retina, in vivo, WT- and P2X7null mice ( $n=6$ at 4,12 , and 18 months) were anesthetized using a mixture of $67 \mathrm{mg} / \mathrm{kg}$ ketamine and $13 \mathrm{mg} / \mathrm{kg}$ xylazine, and the ocular fundus was imaged using a Micron III Retinal Imaging Microscope (Phoenix Research Labs, Pleasanton, CA). ${ }^{22,23}$ Briefly, the corneal reflex of the mice was extinguished by topical application of $0.5 \%$ proparacaine hydrochloride (Alcaine; Alcon Laboratories, Frenchs Forest, NSW, Australia), and the pupils were dilated with topical application of 1\% atropine sulfate (Alcon Laboratories) and $2.5 \%$ phenylephrine hydrochloride (Bausch \& Lomb, Chatswood, NSW, Australia). Mice were placed in a purpose-built holder, and the lens of a Micron III was placed on the cornea using a corneal lubricant at the interface (Genteal; Alcon Laboratories). Fundus images were viewed and collected using specialty Micron III software. Fundus spots were counted using a custom macro in ImageJ version 1.43 (NIH, Bethesda, MD; https://imagej.nih.gov/ij). Specifically, fundus area was manually defined, the image was smoothed to improve edge definition, and the analyze particles tool was used to assess number of spots.

\section{Electroretinography}

The function of the retina was assessed using a twin-flash electroretinogram (ERG) to differentiate rod and cone pathway function in WT- and P2X7-null mice, as previously described. ${ }^{23-25}$ At least $n=10$ mice of each strain at 4, 12, and 18 months were assessed. Briefly, mice were anesthetized as above, the recording electrode was placed on the cornea, and the reference electrode was placed in the mouth. The ERG stimulus and recording were coordinated using Scope software version 3.6.10 (ADInstruments Pty Ltd, Castle Hill, NSW, Australia). ERG responses were amplified (ML132 BioAmp; ADInstruments) and acquired at a 10-kHz sampling frequency over a 250-millisecond epoch (ML785 Powerlab/8sp amplifier; ADInstruments). To elicit the ERG, a 2.1-log cd $\cdot$ second $/ \mathrm{m}^{2}$ full-field flash was delivered using a commercial imaging flash unit via a Ganzfeld custom-made by the authors (Visual Neuroscience Laboratory, Melbourne,
VIC, Australia). Two flashes were delivered with a 0.8-second interstimulus interval, to elicit responses from the rod and cone pathways (mixed response) and the cone pathway (cone response) alone. The cone response was digitally subtracted from the mixed response to generate the rod response. ERG component analysis was completed using previously published equations and techniques. ${ }^{26}$ The rod photoreceptor responses (rod a-wave) were isolated and analyzed using a modified PIII model, to derive the amplitude of the PIII response (PIII Rmax in $\mu \mathrm{V}$ ) and the sensitivity $\left(\mathrm{S}\right.$ in $\left.\mathrm{m}^{2} \mathrm{~cd}^{-1} \mathrm{~s}^{-3}\right)$. The rod and cone post-photoreceptoral function (b-wave) was isolated and then fitted using an inverted $\gamma$ function to generate the PII. From the PII fit, the amplitude of the PII response (PII amplitude in microvolts) and the time to peak (time in milliseconds) were derived.

\section{Phagocytosis Assay}

To assess for changes in blood-borne macrophage and retinal microglia/infiltrating macrophage phagocytosis, flow cytometry techniques were used, as described previously. ${ }^{17,20,27}$ Retinal and blood samples from two mice were pooled for each age to generate at least $n=5$ samples from each genotype at 4, 12, and 18 months. Mice were deeply anesthetized with $200 \mathrm{mg} / \mathrm{kg}$ ketamine and $40 \mathrm{mg} / \mathrm{kg}$ xylazine. Blood samples were collected by cardiac puncture into heparinized tubes. The red blood cells were lysed, and the white blood cells were centrifuged $(400 \times g)$, washed, and placed in fluorescence-activated cell sorting buffer, as previously described. ${ }^{17}$ At the same time, retinal samples were collected and dissociated using an enzymatic digest (Neural Dissociation kit; Miltenyi-Biotec, Macquarie Park, NSW, Australia), according to the manufacturer's instructions, centrifuged $(400 \times g)$, washed, and placed in fluorescenceactivated cell sorting buffer. Both retina and blood samples were then incubated in CD11b-allophycocyanin-conjugated antibody (1:10; catalog number 130-098-088; clone M1/ 70.15.11.5; Miltenyi-Biotec) for 15 minutes at $4^{\circ} \mathrm{C}$, washed, and placed in $4 \mathrm{~mL}$ of fluorescence-activated cell sorting buffer on ice for analysis. In mouse, CD11b labels macrophages as well as neutrophils, natural killer cells, granulocytes, and $\mathrm{CD}^{+}$B-1 cells. Right Reference Standard Fluorescein High beads (Polysciences, Warrington, PA) were used to calibrate the flow cytometer daily (FACSCalibur flow cytometer; BD Biosciences, San Jose, CA). Flow cytometry was then used to assess baseline fluorescence in the far red and green channels for each sample before analysis of phagocytosis, which was quantified by addition of Fluoresbrite yellow green carboxylate latex microspheres (1- $\mu \mathrm{m}$ YG beads; Polysciences). Data were collected over 7 minutes. The linear mean channel of fluorescence intensity for each gated subpopulation over successive 10-second intervals was analyzed by WinMDI software version 2.9 (Dr. Joseph Trotter, Scripps Institute, La Jolla, CA) and plotted against time. A kinetics plot of YG-bead uptake was fit using a one-phase exponential equation (GraphPad Software, Inc., 
La Jolla, CA). The rate (K) of macrophage uptake of YGbeads was assessed for each sample. In some preliminary experiments, $20 \mu \mathrm{mol} / \mathrm{L}$ cytochalasin $\mathrm{D}$ was added to distinguish YG-bead adhesion from phagocytosis and 1 mmol/L ATP was added to confirm P2X7-mediated phagocytosis (data not shown), confirming that macrophages in the blood and retina of WT and P2X7-null mice perform consistently with previously published findings. ${ }^{17,20,27}$ As sample volume was limited, for comparison across genotype and age, only general macrophage uptake of YG-beads was able to be assessed.

\section{RNA Isolation and Quantitative PCR}

For gene expression analysis, peripheral blood was isolated from 4-month-old WT and P2X7-null animals ( $n=8$ in each group). Total RNA was isolated from the blood samples using commercial spin columns (QIAamp RNA Blood Minikit; Qiagen, Melbourne, VIC, Australia). Mmp2, Mmp3, and Mmp9 gene expression was quantified using real-time quantitative PCR. Total RNA samples (250 ng per sample) were reverse transcribed (Tetro cDNA synthesis kit; Bioline, Sydney, NSW, Australia) and amplified (Rotor-Gene SYBR Green PCR kit; Qiagen) using specific primers [Mmp2, 5'-GTCGCCCCTAAAACAGACAA-3' (forward) and $5^{\prime}$-GGTCTCGATGGTGTTCTGGT-3' (reverse); Mmp3, 5'-CAGACTTGTCCCGTTTCCAT-3' (forward) and 5'-GGTGCTGACTGCATCAAAGA-3' (reverse); Mmp9, 5'-CGTCGTGATCCCCACTTACT-3' (forward) and 5'-AACACACAGGGTTTGCCTTC$3^{\prime}$ (reverse)]. External standards were used for quantification of gene copy number, as previously described, ${ }^{28}$ using the housekeeping genes, hypoxanthine guanine phophoribosyl transferase [Hprt, 5'-CCTAAGATGAGCGCAAGTTGAA-3' (forward) and 5'-CCACAGGACTAGAACACCTGCTAA- $3^{\prime}$ (reverse)] and glyceraldehyde-3-phosphate dehydrogenase [Gapdh, 5'-TGTGTCCGTCGTGGATCTGA-3' (forward) and $5^{\prime}$-TTGCTGTTGAAGTCGCAGGAG-3' (reverse)]. Data are expressed as copies/copy Gapdh.

\section{Histology and Transmission Electron Microscopy}

To assess the structure of the neural retina, RPE, and choroid, histology was undertaken. ${ }^{22,23}$ At least $n=6$ mice of each strain at 4,12 , and 18 months were assessed. For all procedures involving tissue collection, mice were first deeply anesthetized (as above) and euthanized by cervical dislocation. The eyecups were isolated and fixed overnight in $1 \%$ paraformaldehyde, $2.5 \%$ glutaraldehyde, $3 \%$ sucrose, and $0.01 \%$ calcium chloride in $0.1 \mathrm{~mol} / \mathrm{L}$ phosphate buffer $(\mathrm{PB}), \mathrm{pH}$ 7.4. The eyecups were washed in cacodylate buffer, and then incubated in $0.5 \% \mathrm{OsO}_{4}$ before being dehydrated in methanol $(70 \%, 80 \%, 90 \%$, and 100\%) and acetone $(100 \%)$. After embedding in an epoxy resin (ProSciTech, Kirwan, QLD, Australia), blocks were polymerized overnight at $60^{\circ} \mathrm{C}$. For histology, the eyecups were cut into sections ( $1 \mu \mathrm{m}$ thick) on an ultramicrotome (Reichert-
Jung Ultracut S; Reichert, Depew, NY) and stained using $0.5 \%$ toluidine blue. An Axioplan microscope (Carl Zeiss, Göttingen, Germany) was used to view retinal sections $(\times 40$ magnification under oil), and images, selected based on correct eccentricity and good retinal alignment of photoreceptor nuclei and outer segments, were captured using a digital camera and SPOT software version 3.5.2 (Diagnostic Instruments, Victoria Park, WA, Australia). Images were adjusted for white levels, brightness, and contrast with Adobe Photoshop CS4 (Adobe Systems, Inc., San Jose, CA). Segmentation analysis, as is applied to optical coherence tomography images, was used to determine changes in retinal layer thickness using custom macros written in ImageJ and Microsoft Excel 2007 (Microsoft Corp., Redmond, WA). ${ }^{29}$ Data are presented for the central retina, as no effect of eccentricity was observed (data not shown).

To determine the ultrastructure of the RPE and Bruchs membrane, transmission electron microscopy was completed. The method has been described previously. ${ }^{22,30}$ Ultrathin sections (70 $\mathrm{nm}$ thick) were cut on the ultramicrotome, collected on Formvar-coated copper grids, contrasted with uranyl acetate and lead citrate solutions, and viewed with a Phillips CM120 electron microscope (Field Electron and Ion Company, Hillsboro, OR). The Bruchs membrane was imaged at $\times 13,500$ magnification. The Bruchs membrane was classified according to that described by Hogan and Alvarado ${ }^{5}$ as consisting of five layers: basement membrane of the RPE, inner collagen, elastin layer, outer collagen, and basement membrane of the choroid. Deposits, similar in appearance to basal laminar and basal linear deposits, were included in the thickness analysis. Custom segmentation analysis ${ }^{29}$ was used to determine changes in membrane thickness. RPE cells were imaged at $\times 5800$ magnification and i) transverse cell area/length of field, ii) fractional melanosome area $(\%)$, melanosome area $\left(\mathrm{nm}^{2}\right)$ per RPE area $\left(\mathrm{nm}^{2}\right)$, and iii) fractional vacuole area $(\%)$, vacuole area $\left(\mathrm{nm}^{2}\right)$ per RPE area $\left(\mathrm{nm}^{2}\right)$, assessed using a custom ImageJ macro. Specifically, RPE area and field length were manually defined, the image was smoothed to improve edge definition, and the analyze particles tool was used to assess melanosome and vacuole area. At least six images of Bruchs membrane and five images of RPE were analyzed, and the results were averaged to generate a single result $(n=1$ per animal). Data are presented per animal ( $n=6$ per strain per age) and assessed for statistical comparison.

\section{Indirect Immunofluorescence Histochemistry}

Isolated eyecups were fixed in $4 \%$ paraformaldehyde in 0.1 $\mathrm{mol} / \mathrm{L} \mathrm{PB}$ for 30 minutes. The tissue was then washed with PB and cryoprotected by immersion in graded sucrose concentrations, $10 \%$ and $20 \%$ for 1 hour, then $30 \%$ sucrose in PB overnight at $4^{\circ} \mathrm{C}$ before being stored at $-20^{\circ} \mathrm{C}$. For sections, tissues were embedded in OCT compound (Tissue Tek; Sakura Finetek, Tokyo, Japan) before rapid freezing into blocks. Transverse sections of the eyecup were cut at $14 \mu \mathrm{m}$ 
with a Microm HM550 cryostat (Microm Pty Ltd, Walldorf, Germany) at $-20^{\circ} \mathrm{C}$, and placed on Polysine glass slides (Polysine adhesion slides; Thermo Scientific, Scoresby, VIC, Australia) before storage at $-20^{\circ} \mathrm{C}$. For labeling of frozen retinal sections, the slides were thawed and rinsed three times for 10 minutes in PB. Primary antibodies were diluted in 3\% normal goat serum, $1 \%$ bovine serum albumin, and $0.5 \%$ Triton X-100 in PB and were applied to the retinal sections overnight. The primary antibodies used to assess Müller glia were as follows: rabbit anti-glial fibrillary acidic protein (GFAP; Immunogen, bovine spinal cord GFAP; catalog number Z0334; Dako, Carpinteria, CA), diluted 1:20,000 to label astrocytes and gliotic Müller cells, and mouse anti-glutamine synthetase (GS; Immunogen, glutamine synthetase purified from sheep brain; clone GS-6; catalog number MAB302; Merck-Millipore, Frenchs Forest, NSW, Australia), diluted 1:1000 to label Müller cells. Antibodies against factors in the complement cascade were also tested using serum-free antibody diluent, including rat anti-C3 [1:100; Immunogen, C57BL/6 thymocytes saturated with rat anti-Thy-1 monoclonal antibody of IgG2b subclass (RmT1), catalog number AB11862; Abcam, Melbourne, VIC, Australia] and mouse anti-C5 (1:100; Immunogen, purified mouse C5, catalog number HM1073; Hycult Biotech, Uden, the Netherlands). Sections were rinsed in PB and incubated with secondary antibody: goat anti-mouse, goat anti-rat, or goat anti-rabbit conjugated to AlexaFluor 488 or AlexaFluor 594 (1:500; Life Technologies, Mulgrave, VIC, Australia), as required, for 90 minutes. Finally, sections were rinsed in PB before coating in a custom mounting media [Mowiol 1\% w/v (polyvinyl alcohol), 25\% glycerol, 2.5\% 1,4-diazabicyclo-(2,2,2)-octane in $0.1 \mathrm{~mol} / \mathrm{L}$ Tris buffer, $\mathrm{pH}$ 7.4], and protected by application of a glass coverslip.

For labeling of RPE flat mounts, eyecups consisting of $\mathrm{RPE}$, choroid, and scleral tissues in $30 \%$ sucrose/PB were snap frozen in liquid nitrogen and thawed three times to enhance antibody penetration and then washed in PB. RPE were then incubated for 4 days in primary antibody at $4^{\circ} \mathrm{C}$ to label microglia and infiltrating macrophages (rabbit antiIbA1; Immunogen, synthetic peptide corresponding to the $\mathrm{C}$ terminus of human IbA1: PTGPPAKKAISELP; 1:1500; catalog number 019-19741; Wako Pure Chemical Industries, Richmond, VA); for tight junctions in the RPE, mouse anti-zonula occludens-1 (ZO-1; 1:200; Immunogen, human recombinant ZO-1 fusion protein encompassing amino acids 334 to 634; clone ZO1-1A12; catalog number 339100; Molecular Probes, Thermofisher Scientific, Melbourne, VIC, Australia) was used. After washing in PB, RPE were incubated overnight with secondary antibodies, as above. The tissues were washed in PB, RPE side up on a slide, coated in a custom glycerol/Mowiol-based mounting media, and covered with a glass coverslip. The method has been described previously. ${ }^{23}$

A Zeiss Meta or Pascal confocal microscope (air, 10× objective; air, $20 \times$ objective; and oil, $40 \times$ objective) was used to examine and image immunolabeled tissue samples
(Carl Zeiss AG, Oberkochen, Germany). In some instances, autofluorescence was assessed in the absence of immunolabeling by increasing the laser power $(30 \%)$ and gain of the confocal settings in the far red (647-nm excitation) spectral frequency. ${ }^{23}$ Images were collected using Zeiss LSM Image Browser 3.2 (Carl Zeiss AG) or ZEN lite software version 2.3 (Carl Zeiss AG). Images were edited using LSM Zeiss software, ImageJ, or Adobe Photoshop CS2. Image quality was optimized similarly for WT and P2X7-null tissues by adjusting the following parameters: laser power, gain, and black levels.

\section{Analysis of RPE Cell Morphology in Flat Mount}

Eyecups from 4- and 18-month-old WT and P2X7-null mice were compared for changes in RPE cell size $(n=6$ mice for each age). Six images (three central and three peripheral) from ZO-1-labeled RPE were collected for each animal using a $20 \times$ air objective. ImageJ 1.43 freeware (NIH) was used to analyze RPE cell area. ${ }^{23}$ For a given RPE, hundreds of cell areas were assessed and averaged to generate a single result ( $n=1$ per animal) for both central and peripheral RPE. Data are presented per animal ( $n=6$ per eccentricity per strain) and assessed for statistical comparison.

\section{Analysis of Müller Cell Gliosis}

Transverse retinal sections labeled with GS for Müller cells and GFAP for gliotic Müller cells from 4-, 12-, and 18month-old WT and P2X7-null mice were compared $(n=6$ mice for each age). For each section, a tile scan image of the entire retina was captured at $\times 20$ magnification and analyzed using a custom ImageJ and Microsoft Excel macro for retinal length, GS profiles, and GFAP/GS profiles at $50 \%$ of depth of the inner plexiform layer. Data are presented as follows: the number of GFAP cells as a percentage of the number of GS cells and the number of GS cells expressed per length of retina $(\mathrm{mm})$. Data are presented for the entire retina, as no effect of eccentricity was observed (data not shown).

\section{Statistical Analysis}

Results are expressed as the means per group \pm SEM. Retinal pigment epithelium cell size and protein expression across eccentricity (central and peripheral) and genotype (WT versus P2X7-null mice) were analyzed by two-way analysis of variance. ERG responses across ages (4, 12, 15, and 18 months) and genotype (WT versus P2X7-null mice) were analyzed by two-way analysis of variance. After twoway analysis of variance, a Tukey post hoc test was used to make individual comparisons, as appropriate (Graphpad Prism version 6; GraphPad Software, Inc., San Diego, CA), and statistical significance between genotypes and age was considered at $P<0.05$. For fundus spot counts and immunohistological quantification of RPE macrophage 
numbers at 18 months, a $t$-test was used to compare results from WT and P2X7-null mice.

\section{Results}

\section{Macrophage Phagocytosis Is Reduced in P2X7-Null Mice and Also with Age in WT Mice}

Macrophages are critical for clearance of debris under normal and pathological situations and may aid the aging RPE by phagocytosing debris from the Bruchs membrane. In line with this suggestion a choroidal, blood-borne monocyte can be seen at the ultrastructural level, extending a process into a region of thickened Bruchs membrane in a 12-month-old WT mouse (Figure 1, A and B). To investigate how age and lack of $\mathrm{P} 2 \mathrm{X} 7$ receptor scavenger function affect this process, we used flow cytometry to assess blood and retinal macrophage phagocytosis in WT and P2X7-null mice. As an example, at 4 months of age in WT mice, blood-borne macrophages showed high affinity for phagocytosis of YG-beads (Figure 1C). Macrophages from P2X7-null blood were slower to engulf the beads, but eventually reached the same plateau, suggesting the presence of functional redundancy, whereby multiple scavenger receptors are involved in phagocytosis. For analysis, the rate of phagocytosis was assessed by fitting an exponential equation to these curves, and this was compared as a function of age for macrophages in the neural retina and also in the peripheral blood. In the retina, the rate of macrophage phagocytosis, which would primarily represent microglial activity, was reduced in P2X7null mice at 4, 12, and 18 months of age (two-way analysis of variance, age $P=0.98$, genotype $P<0.001$, post hoc) (Figure 1D). In the blood, the rate of macrophage phagocytosis was reduced in the WT mice with age between 4 and 18 months, suggesting a reduction of macrophage activity may normally occur with age $(P<0.05)$ (Figure $1 \mathrm{E})$. In the P2X7null mice, blood macrophage phagocytosis was reduced compared to WT at both 4 and 12 months of age $(P<0.05)$ but not at 18 months of age.

Synthesis and secretion of matrix metalloproteinases (MMPs) has been shown to be regulated by P2X7 receptors on macrophages. ${ }^{31}$ As MMPs, in particular MMP-2 and MMP-9, play an important role in proteolytic degradation of type 4 collagen, which is the principal component of basement membranes, we hypothesized that peripheral blood cells might modulate phagocytosis and digestion of Bruchs
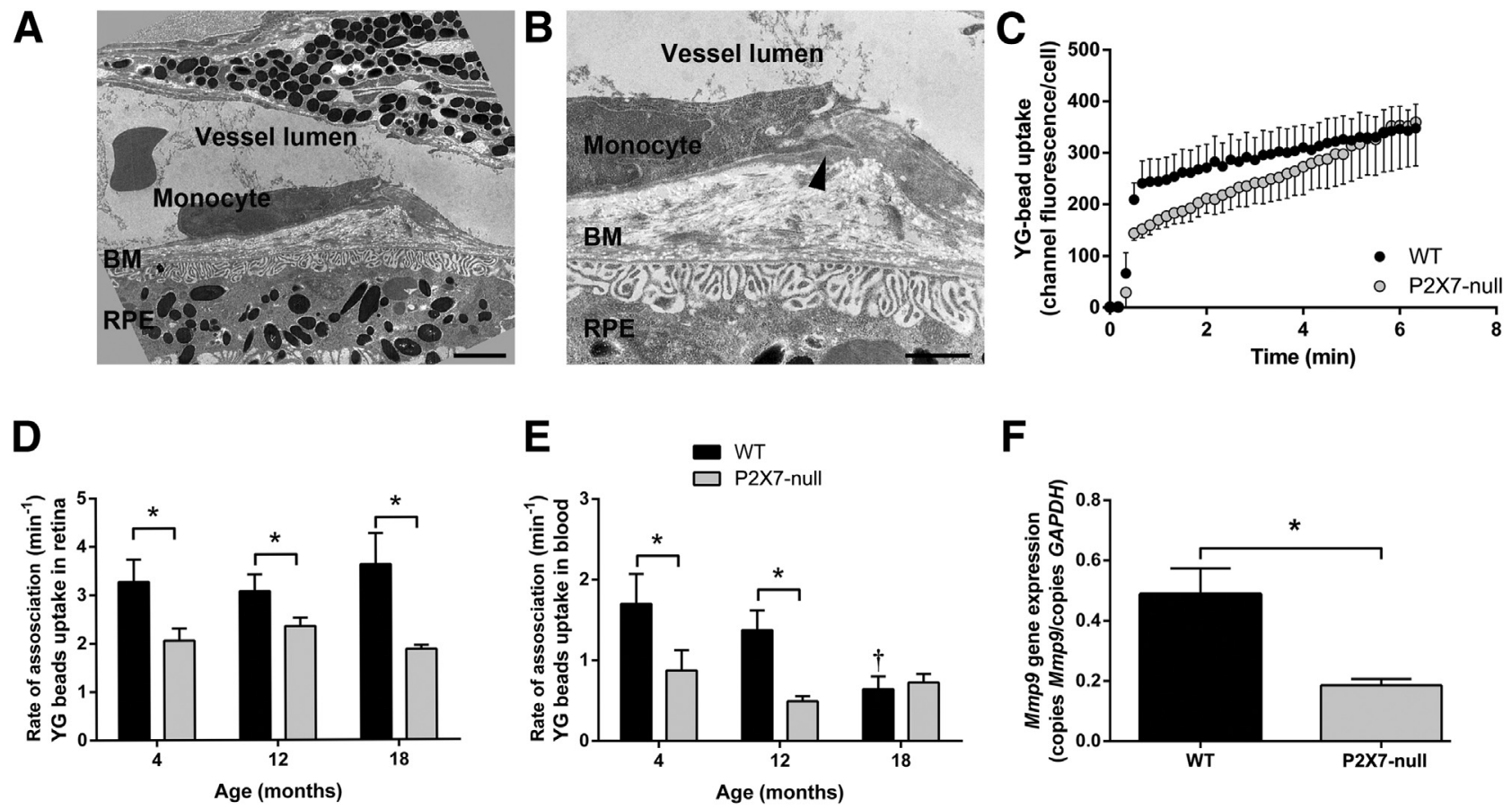

Figure 1 Macrophage phagocytosis is reduced with age in WT mice and also in P2X7-null mice. A: Transmission electron micrograph of Bruchs membrane (BM) in a 12-month-old WT mouse showing a choroidal monocyte extending a process into the BM. B: Magnified view of cell in A, showing a choroidal monocyte process in the BM (arrowhead). C: Flow cytometry was used to assess macrophage phagocytosis of YG-beads. As an example, at 4 months of age in WT mice, blood-borne macrophages show high affinity for phagocytosis of YG-beads, whereas macrophages from P2X7-null blood are slower. The rate of phagocytosis was assessed by fitting an exponential equation to these curves. D: In the retina, the rate of macrophage phagocytosis was reduced in P2X7-null mice at 4, 12, and 18 months of age (two-way analysis of variance, age $P=0.98$, genotype $P<0.001$ ). E: In the blood, the rate of macrophage phagocytosis is reduced in the WT mice with age between 4 and 18 months. In the P2X7-null mice, the rate of macrophage phagocytosis is reduced compared to WT at both 4 and 12 months of age. F: As MMPs play an important role in degradation of collagen, which is the principal component of the BM, we used RT-PCR to quantify Mmp-9 mRNA in blood from P2X7-null mice and found there was significantly less than in WT blood [data are expressed as Mmp9/glyceraldehyde-3-phosphate dehydrogenase (GAPDH) copies]. Data are expressed as means \pm SEM. $n \geq 5$ (C-F). ${ }^{\star} P<0.05$ (post-hoc); ${ }^{\dagger} P<0.05$ versus 4 months (post-hoc). Scale bars: 2 $\mu \mathrm{m}(\mathbf{A}) ; 1 \mu \mathrm{m}$ (B). RPE, retinal pigment epithelium. 
membrane, the RPE/choroidal basement membrane, via release of MMPs. Mmp-2, Mmp-3, and Mmp-9 mRNA was quantified from WT and P2X7-null mice. Levels of Mmp-2 and Mmp-3 were negligible in both WT and P2X7-null samples; however, there was significantly less Mmp-9 mRNA in blood from P2X7-null mice [WT, $0.4884 \pm 0.08526(n=8)$ versus P2X7-null mice, $0.1855 \pm 0.02150(n=8) ;$ Mmp9/glyceraldehyde-3phosphate dehydrogenase copies] (Figure 1F). These data suggest that the immune cells of P2X7-null mice have reduced expression of enzymes critical for digestion of Bruchs membrane and clearance of debris.

\section{Bruchs Membrane Thickens and Retinal Stress 0ccurs by 12 Months of Age in P2X7-Null Mice}

To investigate the effect of lack of P2X7 scavenger receptor function on Bruchs membrane morphology, we investigated Bruchs membrane thickness in WT and P2X7-null mice as a function of age (Figure 2). In WT mice at 4 months, Bruchs membrane had a thin laminated structure (Figure 2A). This layered structure was less apparent in the P2X7-null mice at the same age (Figure 2B). At 18 months, Bruch's membrane appeared thicker in the WT compared to 4 months of age (Figure 2C). At 18 months, Bruchs membrane was almost twice as thick in the P2X7-null mouse with lesions, which appeared similar to basal linear deposits and basal laminar deposits evident, as well as accumulation of electron-lucent profiles of unknown composition (Figure 2D). However, like all mouse models to date, no true basal linear deposits ${ }^{11,32}$ or drusen analogous to sub-RPE deposits in human AMD were observed. Quantitative analysis indicated that, although there was no significant change in Bruchs membrane thickness between WT and P2X7-null mice at 4 months, by 12 months it was significantly thicker in the P2X7-null mice and remained so at 18 months (two-way analysis of variance, age $P<0.05$, genotype $P<0.001$, post hoc) (Figure 2E). There was also a slight increase in
Bruchs membrane thickness in the WT mice between 4 and 18 months of age, which coincides with the reduced bloodborne macrophage phagocytosis in the 18-month-old animals (Figure 1D).

To investigate the effect on retinal morphology and stress, Müller cell gliosis was assessed (Figure 3). In the WT, there was no change in GFAP expression in GS-positive Müller cell processes between 4 (Figure 3A) and 12 months (Figure $3 \mathrm{~B}$ ), however, there was a subtle up-regulation of GFAP in GS-positive Müller cells by 18 months (Figure 3C). In the P2X7-null mice, there was no gliosis apparent at 4 months (Figure 3D); however, by 12 months of age, there was a significant increase in gliosis (Figure 3E) that remained at similar levels at 18 months (Figure 3F). Quantification of the number of gliotic Müller cells (GFAP positive as a percentage of GS-positive cells) showed a significant increase in gliosis with age and in the P2X7-null mice compared to the WT at 12 months (two-way analysis of variance, age $P<0.0001$, genotype $P=0.0071$, post hoc) (Figure 3G). At 18 months, there was no significant difference between WT and P2X7-null mice in the number of gliotic Müller cells (Figure 3G), possibly because there was trend toward a loss of GS-positive Müller glia in the P2X7null mice (two-way analysis of variance, age $P=0.35$, genotype $P=0.0218$; Tukey post hoc, no significant differences between genotype at any age) (Figure $3 \mathrm{H}$ ).

\section{Photoreceptor Function Is Reduced at 18 Months of Age in P2X7-Null Mice, but There Is No Loss of Photoreceptor Layers}

The rod and cone pathways were assessed in the P2X7-null mice as a function of age (Figure 4). Representative rod ERG waveforms are presented for WT and P2X7-null mice in Figure 4A. At 4 and 12 months of age, there was no significant change in the rod or cone ERG parameters measured; however, at 18 months, there was a significant reduction in the rod photoreceptor response amplitude
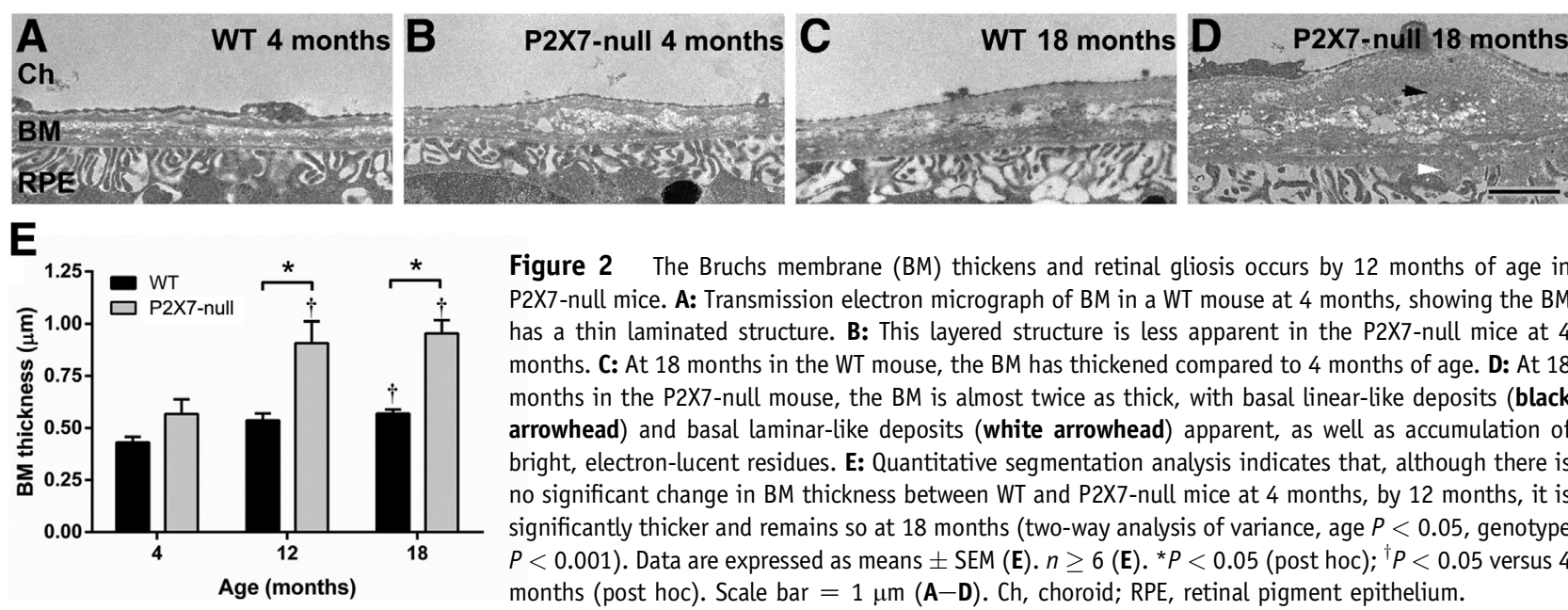

Figure 2 The Bruchs membrane (BM) thickens and retinal gliosis occurs by 12 months of age in P2X7-null mice. A: Transmission electron micrograph of BM in a WT mouse at 4 months, showing the BM has a thin laminated structure. B: This layered structure is less apparent in the P2X7-null mice at 4 months. C: At 18 months in the WT mouse, the BM has thickened compared to 4 months of age. D: At 18 months in the P2X7-null mouse, the BM is almost twice as thick, with basal linear-like deposits (black arrowhead) and basal laminar-like deposits (white arrowhead) apparent, as well as accumulation of bright, electron-lucent residues. E: Quantitative segmentation analysis indicates that, although there is no significant change in BM thickness between WT and P2X7-null mice at 4 months, by 12 months, it is significantly thicker and remains so at 18 months (two-way analysis of variance, age $P<0.05$, genotype $P<0.001$ ). Data are expressed as means \pm SEM (E). $n \geq 6$ (E). ${ }^{*} P<0.05$ (post hoc); ${ }^{\dagger} P<0.05$ versus 4 months (post hoc). Scale bar $=1 \mu \mathrm{m}$ (A-D). Ch, choroid; RPE, retinal pigment epithelium. 

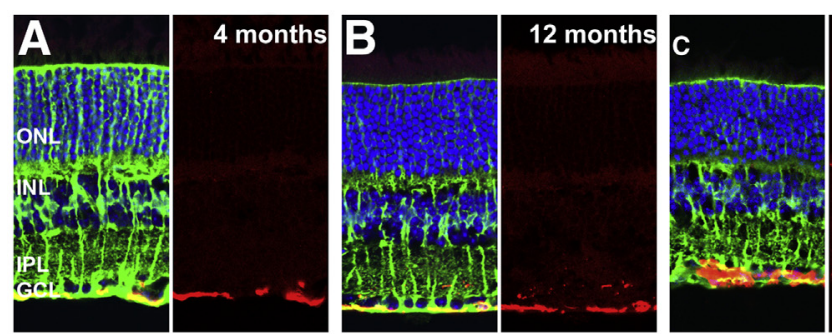

18 month
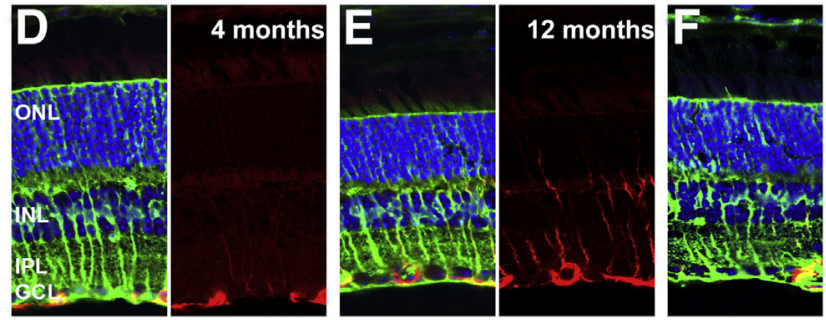

G

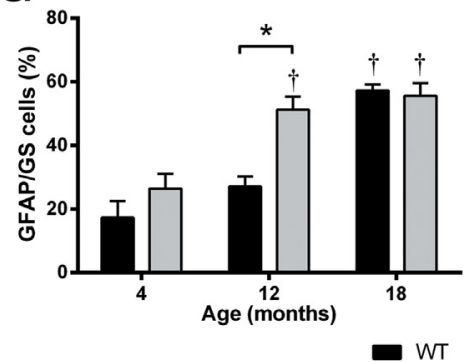

H

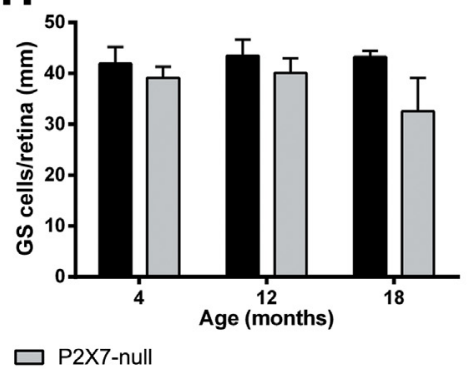

Figure 3 Müller cell gliosis was assessed by labeling for glial fibrillary acidic protein (GFAP) expression (red) in glutamine synthetase (GS) - positive Müller cells (green). A-C: In the WT, there is a subtle up-regulation of GFAP in GS-positive Müller cell processes between 4 (A), 12 (B), and 18 (C) months of age. D-F: In the P2X7-null mice, there is a significant increase in gliosis from 4 (D) to 12 (E) months that remains at similar levels at 18 (F) months and is above that observed for WT. G: Quantification of gliosis as the percentage of GFAP-positive/GS cells shows a significant increase in the percentage of gliotic Müller cells with age and in the P2X7-null mice compared to WT at 12 months (two-way analysis of variance, age $P<0.05$, genotype $P<0.05)$. H: GS-positive Müller cell number is similar in the WT over age; there is a subtle loss of GS-positive cells in the P2X7-null mice (two-way analysis of variance, age $P=0.35$, genotype $P=0.0218$; Tukey post hoc, no significant differences between genotype at any age). Data are expressed as means \pm SEM ( $\mathbf{G}$ and $\mathbf{H}$ ). $n \geq 6$ (G and H). ${ }^{*} P<0.05$ (post hoc); ${ }^{\dagger} P<0.05$ versus 4 months (post hoc). Scale bar $=20 \mu \mathrm{m}(\mathbf{A}-\mathbf{F}) . \mathrm{GCL}$, ganglion cell layer; INL, inner nuclear layer; IPL, inner plexiform layer; ONL, outer nuclear layer.
(Figure 4B) and sensitivity (Figure 4C) in the P2X7-null mice. There was no significant change in the postphotoreceptor responses at this age (data not shown). Representative cone ERG waveforms are presented for WT and P2X7-null mice in Figure 4D. The cone photoreceptor response in rodents is too small to measure, but the postphotoreceptor (b-wave, PII) was subtly affected, with no change in amplitude (Figure 4E), but a slightly slower time to peak (Figure 4F).

Commonly, a diminution of rod photoreceptor function occurs because of a loss of photoreceptors or inner/outer segment length. ${ }^{33}$ To investigate this, histology and retinal layer thickness were examined as a function of age (Figure 5). There were no apparent differences in any of the retinal layers between WT and P2X7-null mice at 4 months (Figure 5, A and B), and although there was a slight decrease in overall retinal thickness with age, there was no difference between WT and P2X7-null mice at 18 months (Figure 5, C and D). The retinal layer thickness was quantified, and there was no significant change in total retinal thickness (Figure 5E), outer nuclear layer thickness (Figure 5F), or inner/outer segment thickness (Figure 5G) between WT and P2X7-null mice. Overall, there were no histological changes in the neural retina, or specifically the photoreceptor layers, at 18 months that might indicate a reason for loss of the rod photoreceptor response amplitude.
RPE Integrity Is Reduced and There Is an Increase in Subretinal Microglia/Macrophages and Inflammatory Markers at 18 Months of Age in P2X7-Null Mice

At 18 months of age, fundus imaging commonly revealed lesions in the P2X7-null mice and to a lesser extent in WT mice (Figure 6, A and B). There were significantly more lesions apparent in the P2X7-null mice than in the WT fundus $(P<0.05)$ (Figure $6 \mathrm{C})$. There were no lesions apparent at earlier ages (data not shown). Given there were no changes in the neural retina that may have contributed to the fundus lesions or ERG deficit, RPE integrity was investigated (Figure 6, D-I). RPE from 4-, 12-, and 18month-old WT and P2X7-null mice were prepared in flat mount and imaged for ZO-1 (green), a tight junction label, and also imaged for autofluorescence (red). There were no changes in RPE structure or autofluorescence apparent at 4 or 12 months; however, at 18 months, when compared with WT (Figure 6D), there was a subtle increase in autofluorescent debris and there were more RPE cells of larger size observed in the P2X7-null eyes (Figure 6E). RPE cell area was quantified, and the RPE cells of P2X7-null mice were significantly larger in both the central and peripheral regions, suggesting loss of RPE cells (two-way analysis of variance, eccentricity $P<0.05$, genotype $P<0.001$ ) (Figure 6F). The RPE of the P2X7-null mice was inspected for multinucleation, a phenomenon of aging that causes 


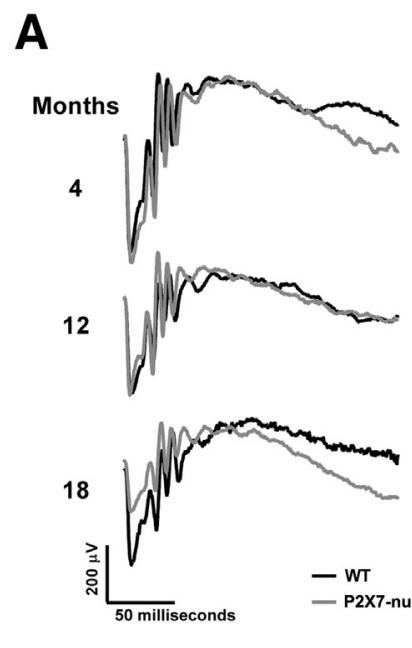

B
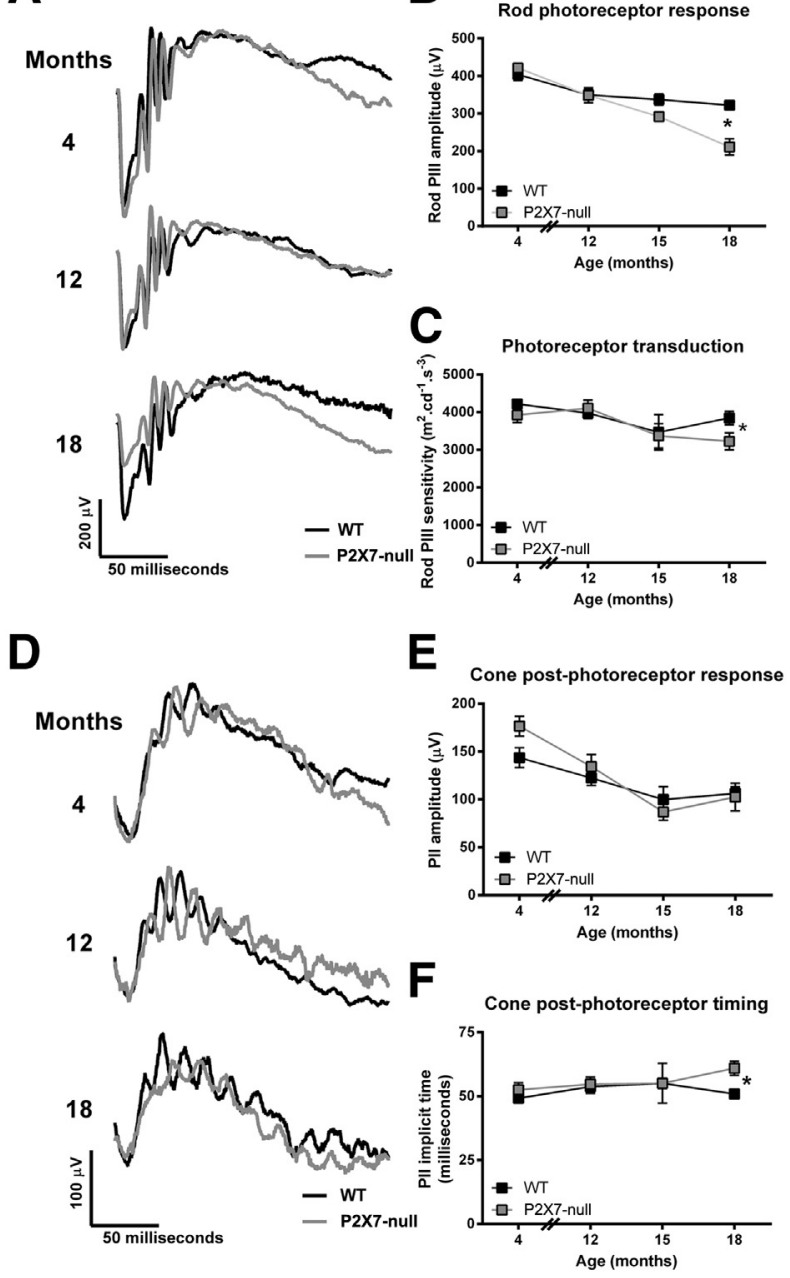

Figure 4 Photoreceptor function is reduced at 18 months of age in P2X7-null mice. The rod and cone pathways were assessed in the P2X7-null mice as a function of age using the electroretinogram (ERG). A: Representative rod ERG waveforms are presented for WT (black line) and P2X7-null mice (gray line) at 4, 12, and 18 months. B and C: At 18 months in the P2X7-null mice, there is a significant reduction in the rod photoreceptor response amplitude (B) and sensitivity (C). D: Representative cone ERG waveforms are presented for WT and P2X7-null mice. E and F: The cone postphotoreceptor (b-wave, PII) is subtly affected with no change in amplitude (E) but a slightly slower time to peak (F). Statistics, two-way analysis of variance, age $P<0.05$, genotype $P<0.001$. Data are expressed as means \pm SEM (B, C, E, and F). $n=5$ (15 months; B, C, E, and F); $n \geq 10$ (all other ages; $\mathbf{B}, \mathbf{C}, \mathbf{E}$, and $\mathbf{F}$ ). ${ }^{*} P<0.05$ WT versus $\mathrm{P} 2 \mathrm{X} 7$ null (post hoc).

cell area increases but is not necessarily related to cell death (Figure 6, G-I). ${ }^{34}$ The larger cells were most commonly binuclear (Figure 6H), as is usual for WT RPE, even at 4 months. Only rarely were RPE cells found to contain more than two nuclei (Figure 6I). This suggests that the increase in cell area observed in the P2X7-null mice may be because of RPE cell loss; however, this cannot be confirmed without analysis of expression of specific cell death markers.

The integrity of the RPE was further interrogated at the ultrastructural level at 4 and 18 months (Figure 7). At 18 months, in the WT eye, the RPE had many melanin granules, some lipofuscin deposits, and few/no vacuoles (Figure 7A). In contrast, the P2X7-null RPE generally appeared altered, with slightly increased lipofuscin content and more vacuoles (Figure 7B). Less commonly, in some regions where Bruchs membrane was significantly thicker, there were large vacuoles and also pigment loss (Figure 7C). Quantitative analysis of the RPE for transverse cell area (Figure 7D), melanosome area (Figure 7E), and vacuole area (Figure 7F) was undertaken. Transverse RPE area per length of field did not change with age or genotype (Figure $7 \mathrm{G}$ ). The fractional area of the RPE covered by melanosomes also did not change with age or genotype (two-way analysis of variance, age $P=0.86$, genotype $P=0.19$ ) (Figure $7 \mathrm{H}$ ). In contrast, the area of the RPE covered by vacuoles increased with age in both WT and P2X7-null mice (two-way analysis of variance; age $P<0.0001$ ) (Figure 7I). In addition, at 18 months, vacuole area was significantly greater in P2X7-null than WT RPE (two-way analysis of variance; genotype $P=0.004$ ) (Figure $7 \mathrm{H}$ ). Together, these findings suggest a reduction of RPE integrity at 18 months may underlie the retinal functional deficits observed.

The RPE of P2X7-null mice was investigated for signs of inflammation. RPE flat mounts were labeled for ZO-1 and the macrophage/microglial marker IbA1 (Figure 8, A-D). In regions where the hexagonal cell structure of the RPE was compromised (Figure 8A), there were many amoeboid, IbA1-positive subretinal macrophages (Figure 8C) that contained large amounts of autofluorescent material (Figure 8B). Although subretinal macrophages are commonly associated with the RPE with increasing age, ${ }^{23}$ they were significantly more apparent in $\mathrm{P} 2 \mathrm{X} 7$-null mice at 18 months $(n=6, P<0.05)$ (Figure $8 \mathrm{E})$. Also, despite the P2X7-null retinal macrophages having a reduced rate of phagocytosis, other scavenger receptor activity must remain as at the ultrastructural level; these subretinal macrophages were found to have phagocytosed RPE-specific melanin granules, even in regions where the RPE appeared relatively healthy (Figure 8, F and G). The subretinal macrophages were further investigated in transverse sections (Figure 8, $\mathrm{H}-\mathrm{M})$. In the P2X7-null mice, cell nuclei found in between the outer segments, adjacent to the RPE (Figure $8 \mathrm{H}$ ), were found to be positive for IbA1 (Figure 8I). Inflammation was investigated by colabeling for IbA1 and the complement markers $\mathrm{C} 3$ and $\mathrm{C} 5$ (Figure 8, $\mathrm{J}-\mathrm{M}$ ). In the WT retina at 18 months, immunofluorescence complement labeling appeared to be primarily associated with the blood vessels and to some extent microglia (Figure 8, $\mathrm{J}$ and L). Labeling with the secondary antibody only (primary antibody omission control) indicated that for both $\mathrm{C} 3$ and $\mathrm{C} 5$, the blood vessel labeling could not be differentiated from secondary antibody recognition of native mouse IgGs in the blood vessels (data not shown). In contrast, in the P2X7-null mice, additional deposition of both C3 (Figure 8K) and C5 (Figure $8 \mathrm{M}$ ) was also apparent between the RPE and photoreceptor outer segments, and was closely associated 

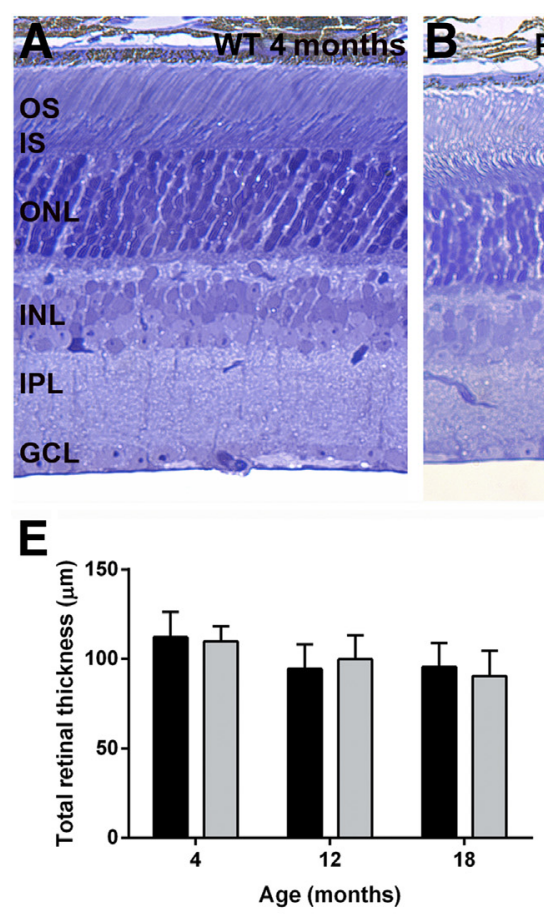
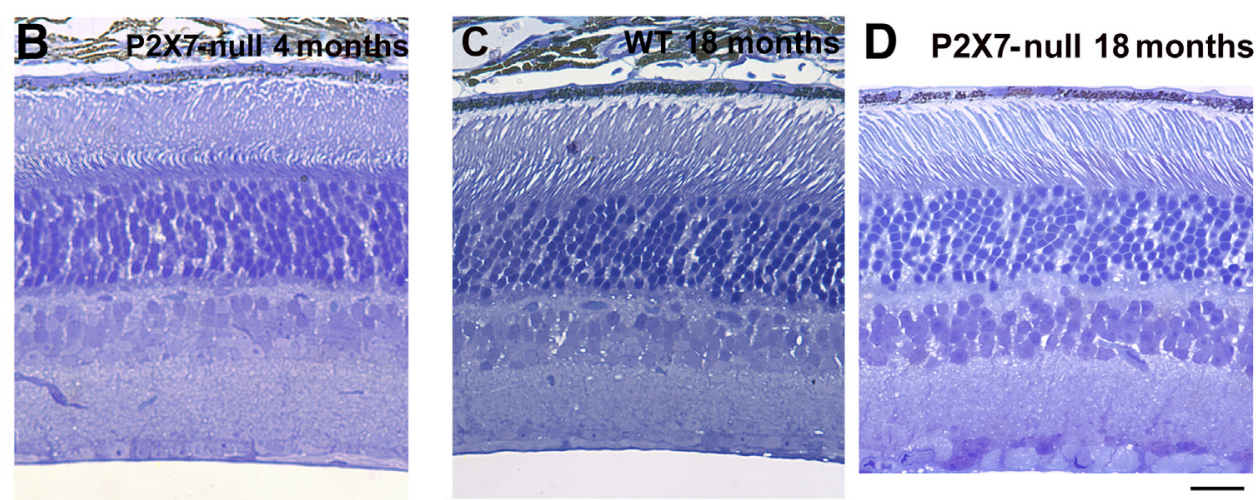

$\mathbf{F}$

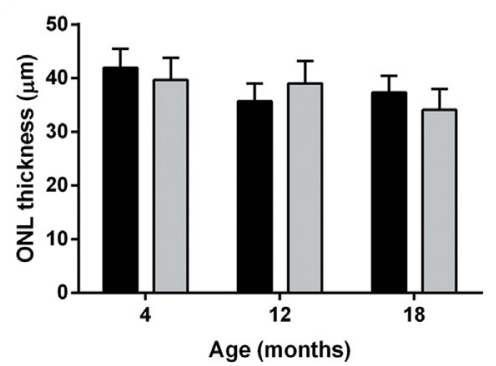

G

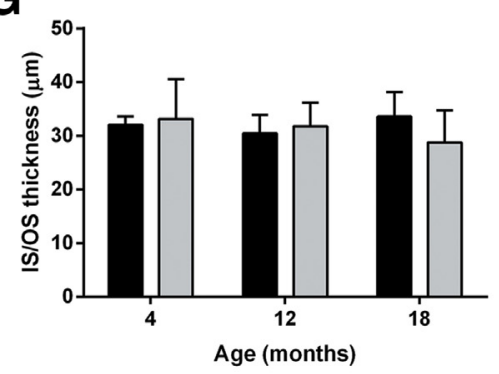

Figure 5 In P2X7-null mice, retinal structure is similar to WT mice at 18 months of age. Histology and retinal layer thickness were examined as a function of age using toluidine blue-stained resin sections (1 $\mu \mathrm{m}$ thick). A and B: Retinal structure appears similar between WT (A) and P2X7-null (B) mice at 4 months. C and D: Similarly, at 18 months, there is no difference between WT (C) and P2X7-null (D) mice. E-G: The retinal layer thickness was quantified, and there is no significant change in total retinal thickness (E), outer nuclear layer (ONL) thickness (F), or inner/outer segment (IS/0S) thickness (G) between WT and P2X7-null mice. Statistics, two-way analysis of variance, age $P<0.05$, genotype $P>0.05$. Data are expressed as means \pm SEM (E-G). $n \geq 5$ (E, $\mathbf{F}$, and $\mathbf{G}$ ). Scale bar $=20 \mu \mathrm{m}(\mathbf{A}-\mathbf{D})$. GCL, ganglion cell layer; INL, inner nuclear layer; IPL, inner plexiform layer.

with subretinal IbA-positive macrophages. These data suggest that subretinal inflammation may occur as a consequence of accelerated RPE failure in P2X7-null mice.

\section{Discussion}

With age, the innate immune system plays an increasing role in systems maintenance, ${ }^{35}$ and it is likely these mechanisms are important in the pathogenesis of AMD. Our hypothesis was that macrophages in the blood and retina aid the aging RPE by clearing debris around the RPE and from Bruchs membrane ${ }^{17}$ and that reduction in this process in the P2X7null mouse would lead to Bruchs membrane thickening and other signs of early AMD. In line with this, we show that the rate of immune cell phagocytosis is reduced in the P2X7null mice at all ages investigated and that subsequently there is an increase in retinal stress, Bruchs membrane thickening, RPE dysfunction, retinal functional losses, and inflammation in the subretinal space. The aged P2X7-null mouse has many posterior pole changes characteristic of early AMD, providing a valuable model in which to investigate the role of scavenger receptor function in the development of this age-related disease.

Our previous work showed that macrophages in the blood of a cohort of white patients with late-stage AMD had reduced phagocytosis activity because of reduction in the scavenger receptor function of the $\mathrm{P} 2 \mathrm{X} 7$ receptor. ${ }^{17}$ In the current study, we investigated $\mathrm{P} 2 \mathrm{X} 7$ receptor scavenger function in aging WT and P2X7-null mice using flow cytometry to assess blood and retinal macrophage phagocytosis. In the blood, the rate of macrophage phagocytosis was reduced in the WT mice with age between 4 and 18 months, indicating a reduction of blood-borne macrophage scavenger activity normally occurs with age. This is in line with a number of studies that have shown a decrease in phagocytic activity with age. ${ }^{36,37}$ However, contradictory data have been reported, with Aprahamian et $\mathrm{al}^{36}$ finding a decline in neutrophil and not alveolar macrophage phagocytosis with age in a model of lung dysfunction, whereas others have shown no change in phagocytosis mediated by bone marrow-derived macrophages in mice with age. ${ }^{35}$ These differences may represent differences in techniques but also may represent in situ expression status of scavenger receptors on macrophages, dependent on their location within the body. Given there are multiple scavenger receptors, some with different roles and some involved in functional redundancy, it is likely that tissue-specific macrophages may have different complements of scavenger receptors and that this may modulate the phagocytic activity with age. In agreement with this idea, in the current study, there was no change in retinal microglial phagocytosis 

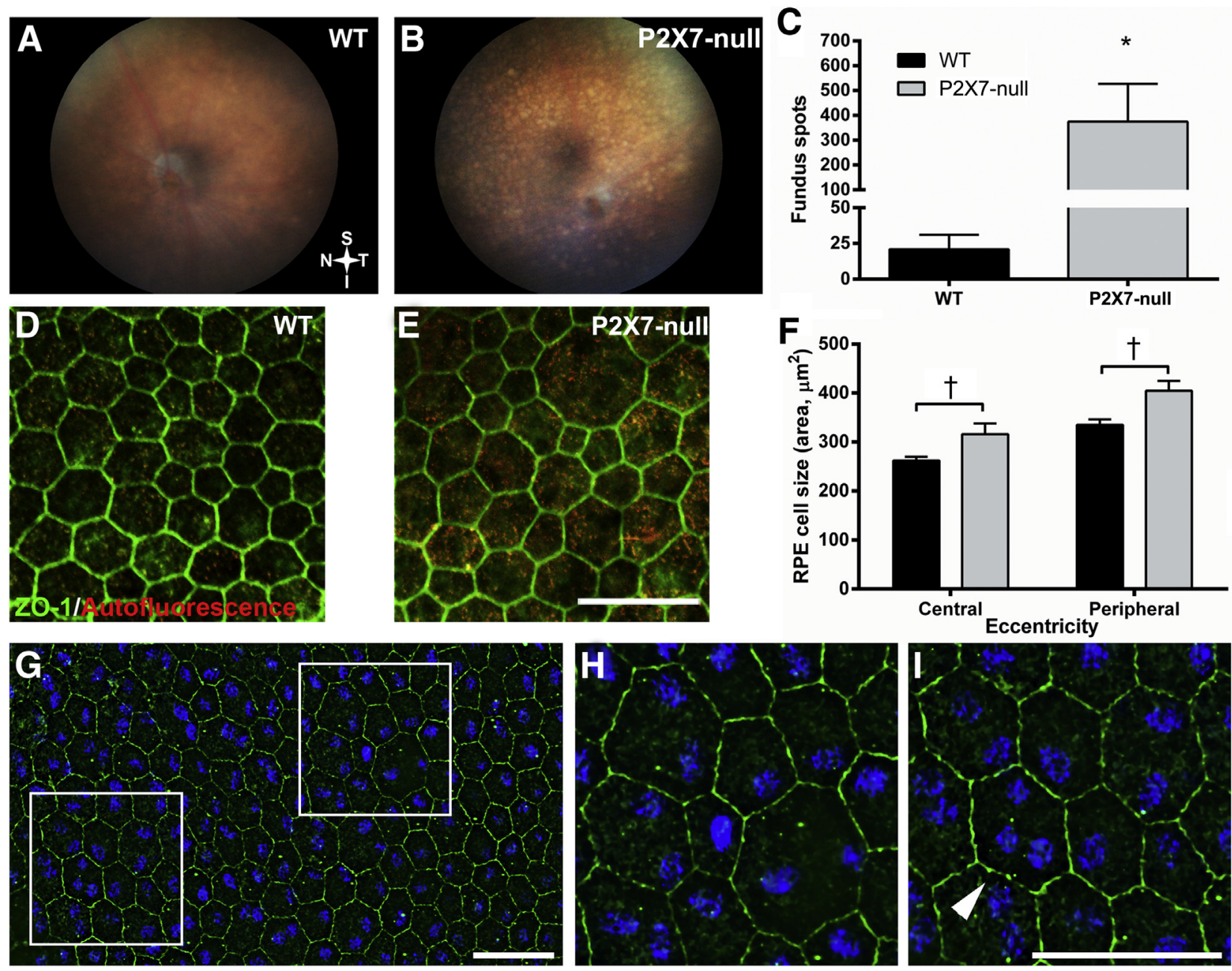

Figure 6 The retinal fundus appears abnormal, and retinal pigment epithelium (RPE) cell size is increased at 18 months of age in P2X7-null mice. A and B: At 18 months, fundus imaging, captured using a Micron III retinal imaging system, commonly reveals lesions in the P2X7-null (A) and to a lesser extent in WT (B) mice. C: There are significantly more lesions apparent in the P2X7-null than in the WT fundus. D and E: Central RPE from 18-month-old WT (D) and P2X7null (E) mice were prepared in flat mount and imaged for zonula occludens-1 (Z0-1; green), a tight junction label, and also imaged for autofluorescence (red). There is a subtle increase in autofluorescent debris and there are more RPE cells of larger size observed in the P2X7-null eyes. F: RPE cells of P2X7-null mice (gray bars) are significantly larger in both the central and peripheral regions than in WTs (black bars), suggesting loss of RPE cells (two-way analysis of variance, eccentricity $P<0.05$, genotype $P<0.001$ ). G-I: The RPE of the P2X7-null mice was inspected for multinucleation by labeling for Z0-1 (green) and cell nuclei, DAPI (blue). H: Right-hand boxed area from G, showing the larger cells in the P2X7-null mice are most commonly binuclear. I: Left-hand boxed area from $\mathbf{G}$, showing a rare example of an RPE cell found to contain more than two nuclei (arrowhead). Data are expressed as means \pm SEM (F). $n \geq 6$ (C and F). ${ }^{*} P<0.05$ versus control ( $t$-test) ${ }^{\dagger} P<0.05$ (post hoc). Scale bars $=50 \mu \mathrm{m}$ (D, E, G, H, and I).

observed in the WT mice with age, suggesting the role these cells play within neural tissues may be different from bloodborne macrophages.

In the P2X7-null mice, blood and retinal macrophage rates of phagocytosis were reduced compared to WT mice from 4 months of age. This is consistent with a role for P2X7 receptor scavenger activity in mice and humans. ${ }^{18,38}$ The reduced scavenger activity in the P2X7-null mouse might contribute to thickening of Bruchs membrane and point to a mechanism of RPE failure with age. In particular, our analysis of mRNA from peripheral blood from P2X7null mice showed reduced Mmp-9 levels, but no change in Mmp-2 or Mmp-3. Indeed, a potential role for MMP-9 in human AMD has recently been identified, as a single-nucleotide polymorphism in this gene was found to be associated with advanced AMD. ${ }^{39}$ Release of MMPs from neutrophils and macrophages allows digestion of collagens in the extracellular matrix, allowing these cell types to move through the interstitial environment. Furthermore, $\mathrm{P} 2 \mathrm{X} 7$ receptor activation has been shown to induce release of MMP-9 from peripheral blood mononuclear cells, and blockade of this receptor reduces release of MMP-9 from these cells. ${ }^{31}$ It is tempting to hypothesize that in the P2X7null mouse, there is failure of peripheral blood macrophages to release Mmp-9 to digest collagens and phagocytose anomalous areas of the Bruchs membrane. In line with this hypothesis, in the P2X7-null mice, there was an increase in Bruchs membrane thickness at 12 months, before significant changes in retinal function and RPE cell loss at 18 months. An increase in Bruchs membrane thickness is a 

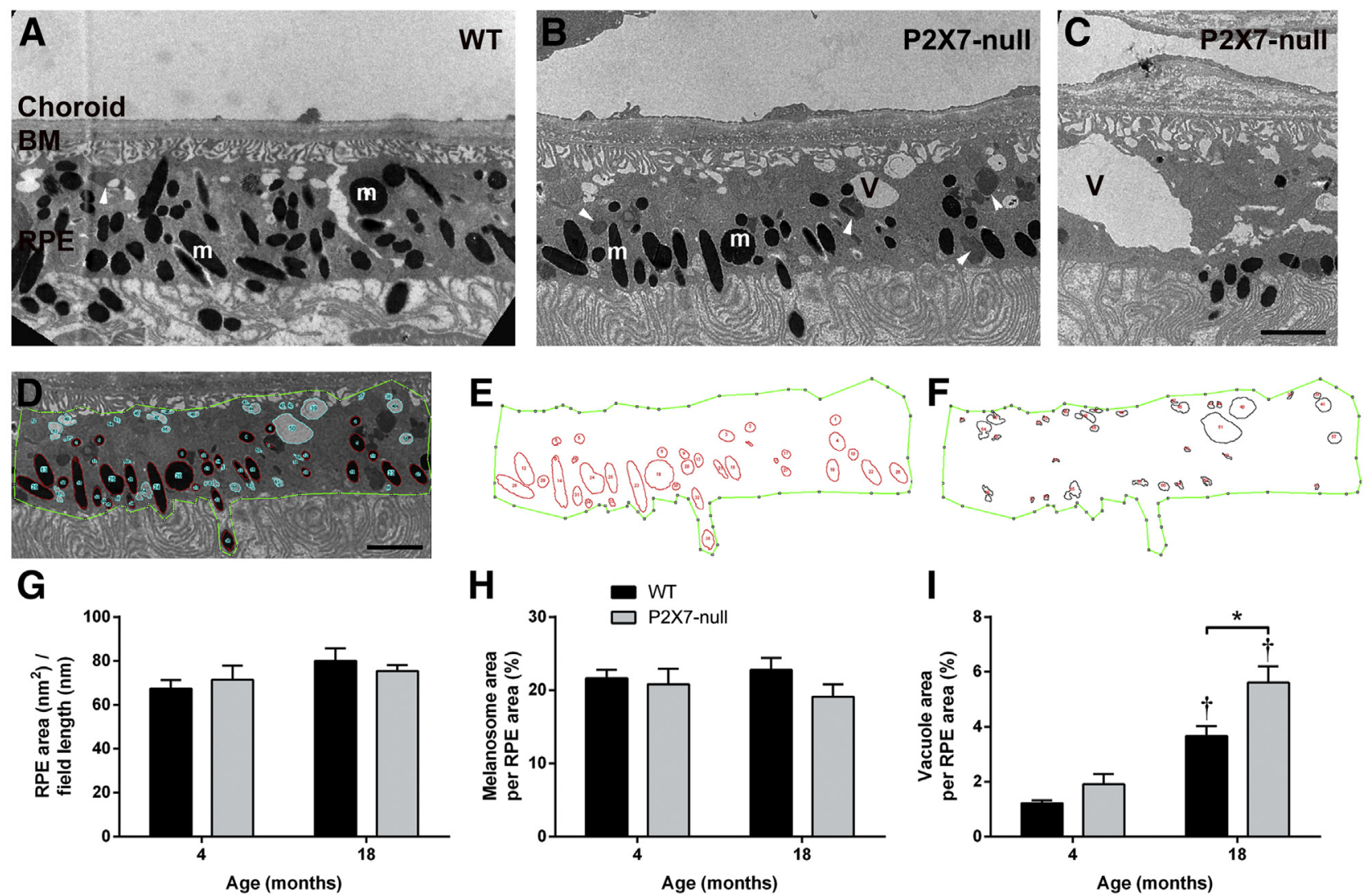

Figure 7 Retinal pigment epithelium (RPE) ultrastructural integrity is reduced at 18 months of age in P2X7-null mice. A-C: Transmission electron micrographs of the RPE at 18 months were assessed. In the WT eye, the RPE has many melanin granules (m), some lipofuscin deposition (white arrowhead), and few/no vacuoles. Note: image has been rotated for consistent orientation, resulting in cropping in the lower left and right corners (black fill) (A). In the P2X7-null mice, there was a slight increase in lipofuscin content (white arrowheads) and more vacuoles (V) (B). Less commonly in the P2X7-null mice, in some regions where Bruchs membrane (BM) is significantly thicker, there are large vacuoles (V) and also pigment loss (C). D-F: Representative RPE image analyzed for RPE cell area/field (green outlines; D), melanosome area (red outlines; E), and vacuole area (black outlines; F) using a custom ImageJ script. G-I: Although RPE area/field $(\mathbf{G})$ and melanosome area $(\mathbf{H})$ do not change with age or genotype, the area of vacuoles increases with age and also in the P2X7-null mice at 18 months (I) (two-way analysis of variance, age $P<0.05$, genotype $P<0.001$ ). Data are expressed as means $\pm \operatorname{SEM}(\mathbf{G}-\mathbf{I}) . n \geq 5$ (G-I). ${ }^{*} P<0.05$ (post hoc); ${ }^{\dagger} P<0.05$ versus 4 months (post hoc). Scale bars $=2 \mu \mathrm{m}(\mathbf{A}-\mathbf{F})$.

characteristic of early AMD in humans ${ }^{6-11}$ and also some previously characterized mouse models, including mice with mutations in lipid transport, apolipoprotein $\mathrm{E}$ null, ${ }^{30}$ mice with a mutation in EFEMP1, which encodes fibulin$3,{ }^{40}$ mice with mutations in tissue inhibitor of metalloproteinases $3,{ }^{41}$ and also mice with mutations in immune system regulation, CD46 null. ${ }^{42}$ In this respect, the P2X7null mouse, which also presents with deposits similar to basal laminar and basal linear deposits, may play a useful role in modeling one of the main pathologies of the early stages of human AMD. ${ }^{4,11}$

In addition to Bruchs membrane thickening, GFAP expression was up-regulated in Müller cells of the retina, suggesting gliosis and retinal stress also occurs at 12 months in P2X7-null mice. Müller cell gliosis occurs with aging, as well as in a range of retinal pathologies, including inner retinal vasculopathies and retinal degenerations. ${ }^{43}$ In humans with AMD, GFAP is up-regulated in Müller cells compared with age-matched tissues. ${ }^{44-46}$ In addition, in AMD eyes, discrete regions of Müller cell gliosis are associated with RPE cell death and drusen. ${ }^{45}$ Thus, Müller cell gliosis may represent an early sign of retinal or RPE dysfunction in the P2X7-null mouse, consistent with findings in AMD patients.

Despite changes in the Bruchs membrane at 12 months, we did not measure significant RPE cell loss and reductions in retinal function in the P2X7-null mouse at this time point. At 18 months of age, functional losses in the photoreceptor response occurred, concomitant with significant RPE cell loss but in the absence of significant histological photoreceptor nuclei or inner/outer segment changes. Commonly, a loss of rod photoreceptor function occurs because of a loss of photoreceptors or inner/outer segment length ${ }^{33}$; however, the present data suggest that, although the photoreceptors remain intact at a histological level, changes in the RPE may contribute to alterations in the visual cycle, leading to photoreceptor functional deficits. The losses in rod photoreceptor function that occur at the same time as RPE deficiencies in the P2X7-null mice are consistent with similar findings in humans, who, although with normal aging show 

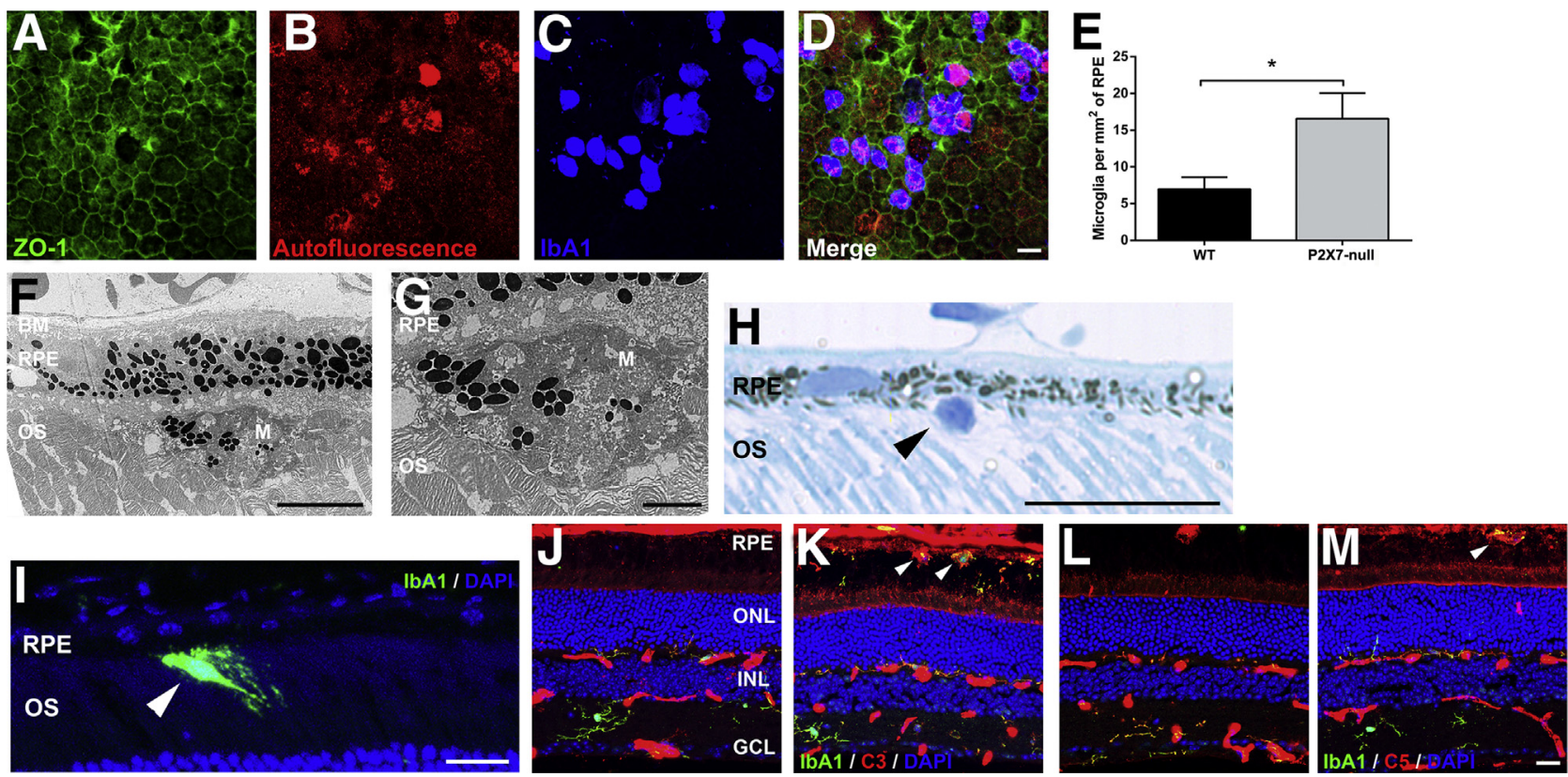

Figure 8 Subretinal inflammation occurs at 18 months of age in P2X7-null mice. A-D: Retinal pigment epithelium (RPE) flat mounts from 18-month-old P2X7-null mice were labeled and imaged for zonula occludens-1 (Z0-1; green; A), autofluorescence (red; B), and the microglial/macrophage marker IbA1 (blue; C); a merge of these images is shown (D). E: There are significantly more IbA1-positive macrophages apparent on the RPE of P2X7-null mice (gray bars) than in WT (black bars) at 18 months. F and G: Transmission electron microscopy of a P2X7-null RPE at 18 months, showing a subretinal macrophage (M), found to contain RPE-specific melanin granules, in regions where the RPE appears relatively healthy ( $\mathbf{F}$, and higher magnification in $\mathbf{G})$. $\mathbf{H}-\mathbf{M}$ : Transverse sections of eyecup. H: Toluidine blue-labeled retinal section, showing an abnormal subretinal cell nuclei (black arrowhead) located within the outer segments (0Ss) adjacent to the RPE in an 18-month-old P2X7-null retina. I: Subretinal cells (white arrowhead) are positive for IbA1 (green); nuclei labeled with DAPI (blue). J-M: Inflammation was investigated by labeling for IbA1 (green), complement markers, C3 and C5 (red), and cell nuclei (DAPI, blue). In the WT retina at 18 months, C3 (J) and C5 (L) are associated with the blood vessels and microglia. In the P2X7-null mice, deposition of both C3 (K) and C5 (M) was also apparent between the RPE and photoreceptor outer segments, and was closely associated with subretinal IbA-positive macrophages (white arrowheads). Data are expressed as means \pm SEM (E). $n \geq 5$ (E). ${ }^{*} P<0.05$. Scale bars: $20 \mu \mathrm{m}(\mathbf{A}-\mathbf{D}$ and $\mathbf{H}-\mathbf{M}) ; 5 \mu \mathrm{m}(\mathbf{F}) ; 2 \mu \mathrm{m}(\mathbf{G})$. BM, Bruchs membrane; GCL, ganglion cell layer; INL, inner nuclear layer; $0 N L$, outer nuclear layer.

loss of scotopic sensitivity and dark adaptation, ${ }^{47}$ have a significantly greater loss of function in the early stages of AMD. $^{48-50}$

At the level of the RPE, aged P2X7-null mice had increased fundus lesions, increased subretinal microglia/ macrophages, an increase in RPE vacuoles, an increase in RPE autofluorescence, and a significant increase in RPE cell area compared with WT controls. Although the increased presence of vacuoles seems to be a common feature of RPE change in mouse models, ${ }^{51-53}$ this is less common in the RPE of humans with AMD. ${ }^{54}$ The source of the fundus lesions in mouse models is also a source of debate. In some studies, the fundus lesions seen on ocular fundoscopy in mice have been suggested to be bloated macrophages/ microglia trapped in the subretinal space. ${ }^{53,55}$ However, our previous study, carefully reanalyzing one of these mouse models, the chemokine (C-C) ligand $2^{-1-}$ mouse, indicates that fundus lesions do not consistently correlate with subretinal macrophages/microglia. ${ }^{23}$ The exact morphological basis underlying the fundus lesions may instead result from a combination of factors, including subretinal macrophages/ microglia, but also relating to RPE dysfunction. First, there was a significant increase in vacuole area in the P2X7-null RPE with age, which may contribute to the appearance of reflective lesions on fundus imaging. Second, there were higher levels of autofluorescence across the RPE, suggestive of increased lipofuscin deposition, A2E, and melanolipofucin accumulation within the RPE, similar to that reported in the $\mathrm{Abca}^{-1-}$ mouse. ${ }^{5-58}$ In addition, subretinal microglia/macrophages would also contribute as autofluorescence was densely associated with these cells in the P2X7-null mice. It is likely that a combination of these autofluorescent macrophages/microglia and other independent regions of RPE autofluorescence and/or vacuolization combine to produce the lesions observed on fundoscopy.

Chronic inflammation, induced by RPE and photoreceptor dysfunction, is thought to play a role in the pathogenesis of many retinal diseases. The accumulation of immune cells at the subretinal space is likely to be a normal aspect of aging, as IbA1-positive cells were observed on the RPE in 18-month-old WT controls, as well as in P2X7-null mice. It is likely that normally retinal microglia/macrophages aid the aging RPE in the clearance of photoreceptor debris and damaged RPE cells from within the subretinal space. ${ }^{16,23,59}$ However, in the P2X7-null mouse, there was a significant increase in the number of subretinal microglia/ macrophages and complement deposition was also apparent. This is similar to the aged chemokine (C-C) ligand 
$2^{-/-}$mice in which subretinal macrophages were also found to be increased after RPE cell loss, but before photoreceptor death. ${ }^{23,53}$ In humans, subretinal macrophages are often associated with subretinal deposits ${ }^{46}$ and have been suggested to potentiate photoreceptor degeneration by inducing an inflammatory response. ${ }^{15,60}$ Some studies have suggested that they contribute to photoreceptor degeneration ${ }^{61-63}$ and the development of drusen. ${ }^{64,65} \mathrm{We}$ did not find evidence for this, as there was no photoreceptor loss despite the increased presence of subretinal macrophages. Rather, the increase in immune cells in the subretinal space of P2X7null mice may be acting as support for a dysfunctional and dying RPE.

Overall, the finding that lack of $\mathrm{P} 2 \mathrm{X} 7$ receptor function induces an early AMD phenotype in mice may be considered counterintuitive, given the body of evidence for a protective role for $\mathrm{P} 2 \mathrm{X} 7$ inhibition in other models of AMD. ${ }^{66-68}$ Although some studies suggest that the RPE expresses P2X7 receptors, ${ }^{69,70}$ we have been unable to detect either mRNA or protein for this receptor in freshly isolated, native mouse RPE or primary (nonpassaged) RPE cell cultures (Supplemental Figure S1). ${ }^{17,30,71,72}$ However, we cannot rule out the possibility that RPE cells express $\mathrm{P} 2 \mathrm{X} 7$ receptors and loss of $\mathrm{P} 2 \mathrm{X} 7$ receptor function may contribute to the phenotype observed in the P2X7-null mouse. In addition, P2X7 receptor-mediated scavenger activity, as described in the current study, is distinct from the more commonly known role for the $\mathrm{P} 2 \mathrm{X} 7$ receptor in release of inflammatory cytokines. ${ }^{18,21}$ When large amounts of extracellular ATP are released, as would occur during rampant cell death, innate phagocytosis activity is halted and the ligand-gated $\mathrm{P} 2 \mathrm{X} 7$ receptor channel is activated, causing inflammasome activation and release of IL- $\beta$ and tumor necrosis factor- $\alpha$ from tissue macrophages. ${ }^{19,20,31,73}$ This highlights that further research is required to determine how the beneficial and detrimental aspects of targeting the $\mathrm{P} 2 \mathrm{X} 7$ receptor can be harnessed for treatment of neurodegenerative diseases and AMD.

In summary, P2X7-null mice display phenotypic characteristics consistent with early AMD, including Bruchs membrane thickening, RPE cell loss, retinal functional deficits, and signs of subretinal inflammation at 18 months of age. These changes may occur because of loss of P2X7 receptor scavenger activity on macrophages, mimicking the process that occurs in a subset of patients with AMD. As this is consistent with our recent findings in human patients with AMD,${ }^{17}$ these mice may provide a useful model for assessing the role of the innate immune system and in particular scavenger receptor function in the development of AMD. Overall, our current data support the hypothesis that scavenger receptor activity on macrophages is required to provide an additional mechanism to aid an aging RPE and that when macrophage scavenger activity is reduced, there is ongoing debris accumulation, Bruchs membrane thickening, and RPE loss. This implicates alterations in the innate immune system and in particular scavenger receptors as a potential contributing cause of AMD.

\section{Supplemental Data}

Supplemental material for this article can be found at http://dx.doi.org/10.1016/j.ajpath.2017.04.016.

\section{References}

1. Lim LS, Mitchell P, Seddon JM, Holz FG, Wong TY: Age-related macular degeneration. Lancet 2012, 379:1728-1738

2. Fritsche LG, Fariss RN, Stambolian D, Abecasis GR, Curcio CA, Swaroop A: Age-related macular degeneration: genetics and biology coming together. Annu Rev Genomics Hum Genet 2014, 15: $151-171$

3. Pikuleva IA, Curcio CA: Cholesterol in the retina: the best is yet to come. Prog Retin Eye Res 2014, 41:64-89

4. Booij JC, Baas DC, Beisekeeva J, Gorgels TGMF, Bergen AAB: The dynamic nature of Bruch's membrane. Prog Retin Eye Res 2010, 29: $1-18$

5. Hogan MJ, Alvarado J: Studies on human macula, 4: aging changes in Bruchs membrane. Arch Ophthalmol 1967, 77:410-420

6. Sarks SH: Aging and degeneration in macular region: clinicopathological study. Br J Ophthalmol 1976, 60:324-341

7. Hogan MJ: Role of the retinal pigment epithelium in macular disease. Trans Am Acad Ophthalmol Otolaryngol 1972, 76:64-80

8. Kliffen M, de Jong PT, Luider TM: Protein analysis of human maculae in relation to age-related maculopathy. Lab Invest 1995, 73: $267-272$

9. Kliffen M, van der Schaft TL, Mooy CM, de Jong PT: Morphologic changes in age-related maculopathy. Microsc Res Tech 1997, 36: $106-122$

10. Reale E, Groos S, Eckardt U, Eckardt C, Luciano L: New components of "basal laminar deposits" in age-related macular degeneration. Cells Tissues Organs 2009, 190:170-181

11. Curcio CA, Millican CL: Basal linear deposit and large drusen are specific for early age-related maculopathy. Arch Ophthalmol 1999, 117:329-339

12. Guymer R, Luthert P, Bird A: Changes in Bruch's membrane and related structures with age. Prog Retin Eye Res 1999, 18: 59-90

13. Strauss O: The retinal pigment epithelium in visual function. Physiol Rev 2005, 85:845-881

14. Feeney L: The phagolysosomal system of the pigment epithelium: a key to retinal disease. Invest Ophthalmol 1973, 12:635-638

15. Buschini E, Piras A, Nuzzi R, Vercelli A: Age related macular degeneration and drusen: neuroinflammation in the retina. Prog Neurobiol 2011, 95:14-25

16. Neumann H, Kotter MR, Franklin RJ: Debris clearance by microglia: an essential link between degeneration and regeneration. Brain 2009 , $132: 288-295$

17. Gu BJ, Baird PN, Vessey KA, Skarratt KK, Fletcher EL, Fuller SJ, Richardson AJ, Guymer RH, Wiley JS: A rare functional haplotype of the P2RX4 and P2RX7 genes leads to loss of innate phagocytosis and confers increased risk of age-related macular degeneration. FASEB J 2013, 27:1479-1487

18. Wiley JS, Sluyter R, Gu BJ, Stokes L, Fuller SJ: The human P2X7 receptor and its role in innate immunity. Tissue Antigens 2011, 78: $321-332$

19. Gu BJ, Rathsam C, Stokes L, McGeachie AB, Wiley JS: Extracellular ATP dissociates nonmuscle myosin from P2X(7) complex: this 
dissociation regulates P2X(7) pore formation. Am J Physiol Cell Physiol 2009, 297:C430-C439

20. Gu BJ, Saunders BM, Jursik C, Wiley JS: The P2X7-nonmuscle myosin membrane complex regulates phagocytosis of nonopsonized particles and bacteria by a pathway attenuated by extracellular ATP. Blood 2010, 115:1621-1631

21. Solle M, Labasi J, Perregaux DG, Stam E, Petrushova N, Koller BH, Griffiths RJ, Gabel CA: Altered cytokine production in mice lacking P2X(7) receptors. J Biol Chem 2001, 276:125-132

22. Vessey KA, Greferath U, Jobling AI, Phipps JA, Ho T, Waugh M, Fletcher EL: Ccl2/Cx3cr1 knockout mice have inner retinal dysfunction but are not an accelerated model of AMD. Invest Ophthalmol Vis Sci 2012, 53:7833-7846

23. Vessey KA, Waugh M, Jobling AI, Phipps JA, Ho T, Trogrlic L, Greferath U, Fletcher EL: Assessment of retinal function and morphology in aging Cc12 knockout mice. Invest Ophthalmol Vis Sci 2015, 56:1238-1252

24. Lyubarsky AL, Chen C, Simon MI, Pugh EN Jr: Mice lacking Gprotein receptor kinase 1 have profoundly slowed recovery of conedriven retinal responses. J Neurosci 2000, 20:2209-2217

25. Vessey KA, Wilkinson-Berka JL, Fletcher EL: Characterization of retinal function and glial cell response in a mouse model of oxygeninduced retinopathy. J Comp Neurol 2011, 519:506-527

26. Ho T, Vessey KA, Cappai R, Dinet V, Mascarelli F, Ciccotosto GD, Fletcher EL: Amyloid precursor protein is required for normal function of the rod and cone pathways in the mouse retina. PLoS One 2012, 7:e29892

27. Wiley JS, Gu BJ: A new role for the P2X7 receptor: a scavenger receptor for bacteria and apoptotic cells in the absence of serum and extracellular ATP. Purinergic Signal 2012, 8:579-586

28. Jobling AI, Vessey KA, Waugh M, Mills SA, Fletcher EL: A naturally occurring mouse model of achromatopsia: characterization of the mutation in cone transducin and subsequent retinal phenotype. Invest Ophthalmol Vis Sci 2013, 54:3350-3359

29. Aplin FP, Luu CD, Vessey KA, Guymer RH, Shepherd RK, Fletcher EL: ATP-induced photoreceptor death in a feline model of retinal degeneration. Invest Ophthalmol Vis Sci 2014, 55:8319-8329

30. Jobling AI, Guymer RH, Vessey KA, Greferath U, Mills SA, Brassington KH, Luu CD, Aung KZ, Trogrlic L, Plunkett M, Fletcher EL: Nanosecond laser therapy reverses pathologic and molecular changes in age-related macular degeneration without retinal damage. FASEB J 2015, 29:696-710

31. Gu BJ, Wiley JS: Rapid ATP-induced release of matrix metalloproteinase 9 is mediated by the P2X7 receptor. Blood 2006, 107: 4946-4953

32. Curcio CA, Johnson M, Rudolf M, Huang JD: The oil spill in ageing Bruch membrane. Br J Ophthalmol 2011, 95:1638-1645

33. Weymouth AE, Vingrys AJ: Rodent electroretinography: methods for extraction and interpretation of rod and cone responses. Prog Retin Eye Res 2008, 27:1-44

34. Chen M, Rajapakse D, Fraczek M, Luo C, Forrester JV, Xu HP: Retinal pigment epithelial cell multinucleation in the aging eye: a mechanism to repair damage and maintain homoeostasis. Aging Cell 2016, 15:436-445

35. Linehan E, Fitzgerald DC: Ageing and the immune system: focus on macrophages. Eur J Microbiol Immunol (Bp) 2015, 5:14-24

36. Aprahamian T, Takemura Y, Goukassian D, Walsh K: Ageing is associated with diminished apoptotic cell clearance in vivo. Clin Exp Immunol 2008, 152:448-455

37. De La Fuente M: Changes in the macrophage function with aging. Comp Biochem Physiol A Comp Physiol 1985, 81:935-938

38. Worthington RA, Smart ML, Gu BJ, Williams DA, Petrou S, Wiley JS, Barden JA: Point mutations confer loss of ATP-induced human P2X(7) receptor function. FEBS Lett 2002, 512:43-46

39. Fritsche LG, Igl W, Bailey JN, Grassmann F, Sengupta S, BraggGresham JL, Burdon KP, Hebbring SJ, Wen C, Gorski M, Kim IK, Cho D, Zhan X, Kwong AM, Boleda A, Brooks M, Gieser L,
Ratnapriya R, Branham KE, Foerster JR, Stambolian D, Haines JL, Iyengar SK, Weber BH, Abecasis GR, Heid IM: A large genome-wide association study of age-related macular degeneration highlights contributions of rare and common variants. Nat Genet 2016, 48:134-143

40. Marmorstein LY, McLaughlin PJ, Peachey NS, Sasaki T, Marmorstein AD: Formation and progression of sub-retinal pigment epithelium deposits in Efemp1 mutation knock-in mice: a model for the early pathogenic course of macular degeneration. Hum Mol Genet 2007, 16:2423-2432

41. Weber BHF, Lin BY, White K, Kohler K, Soboleva G, Herterich S, Seeliger MW, Jaissle GB, Grimm C, Reme C, Wenzel A, Asan E, Schrewe H: A mouse model for Sorsby fundus dystrophy. Invest Ophthalmol Vis Sci 2002, 43:2732-2740

42. Lyzogubov VV, Bora PS, Wu X, Horn LE, de Roque R, Rudolf XV, Atkinson JP, Bora NS: The complement regulatory protein CD46 deficient mouse spontaneously develops dry-type age-related macular degeneration-like phenotype. Am J Pathol 2016, 186: 2088-2104

43. Bringmann A, Pannicke T, Grosche J, Francke M, Wiedemann P, Skatchkov SN, Osborne NN, Reichenbach A: Muller cells in the healthy and diseased retina. Prog Retin Eye Res 2006, 25:397-424

44. Guidry C, Medeiros NE, Curcio CA: Phenotypic variation of retinal pigment epithelium in age-related macular degeneration. Invest Ophthalmol Vis Sci 2002, 43:267-273

45. Wu KH, Madigan MC, Billson FA, Penfold PL: Differential expression of GFAP in early v late AMD: a quantitative analysis. Br J Ophthalmol 2003, 87:1159-1166

46. Greferath U, Guymer RH, Vessey KA, Brassington K, Fletcher EL: Correlation of histologic features with in vivo imaging of reticular pseudodrusen. Ophthalmology 2016, 123:1320-1331

47. Jackson GR, Owsley C, McGwin G Jr: Aging and dark adaptation. Vision Res 1999, 39:3975-3982

48. Dimitrov PN, Guymer RH, Zele AJ, Anderson AJ, Vingrys AJ: Measuring rod and cone dynamics in age-related maculopathy. Invest Ophthalmol Vis Sci 2008, 49:55-65

49. Steinmetz RL, Haimovici R, Jubb C, Fitzke FW, Bird AC: Symptomatic abnormalities of dark adaptation in patients with age-related Bruch's membrane change. Br J Ophthalmol 1993, 77:549-554

50. Owsley C, McGwin G, Clark ME, Jackson GR, Callahan MA, Kline LB, Witherspoon CD, Curcio CA: Delayed rod-mediated dark adaptation is a functional biomarker for incident early age-related macular degeneration. Ophthalmology 2016, 123:344-351

51. Huang H, Liu Y, Wang L, Li W: Age-related macular degeneration phenotypes are associated with increased tumor necrosis-alpha and subretinal immune cells in aged Cxcr5 knockout mice. PLoS One 2017, 12:e0173716

52. Zhao ZY, Chen Y, Wang J, Sternberg P, Freeman ML, Grossniklaus HE, Cai JY: Age-related retinopathy in NRF2-deficient mice. PLoS One 2011, 6:e19456

53. Luhmann UF, Robbie S, Munro PM, Barker SE, Duran Y, Luong V, Fitzke FW, Bainbridge JW, Ali RR, MacLaren RE: The drusenlike phenotype in aging Ccl2-knockout mice is caused by an accelerated accumulation of swollen autofluorescent subretinal macrophages. Invest Ophthalmol Vis Sci 2009, 50:5934-5943

54. Zanzottera EC, Messinger JD, Ach T, Smith RT, Freund KB, Curcio CA: The project MACULA retinal pigment epithelium grading system for histology and optical coherence tomography in age-related macular degeneration. Invest Ophthalmol Vis Sci 2015, 56:3253-3268

55. Chen M, Muckersie E, Forrester JV, Xu H: Immune activation in retinal aging: a gene expression study. Invest Ophthalmol Vis Sci 2010, 51:5888-5896

56. Charbel Issa P, Barnard AR, Singh MS, Carter E, Jiang Z, Radu RA, Schraermeyer U, MacLaren RE: Fundus autofluorescence in the Abca4(-/-) mouse model of Stargardt disease: correlation with accumulation of A2E, retinal function, and histology. Invest Ophthalmol Vis Sci 2013, 54:5602-5612 
57. Keilhauer CN, Delori FC: Near-infrared autofluorescence imaging of the fundus: visualization of ocular melanin. Invest Ophthalmol Vis Sci 2006, 47:3556-3564

58. Sparrow JR, Blonska A, Flynn E, Duncker T, Greenberg JP, Secondi R, Ueda K, Delori FC: Quantitative fundus autofluorescence in mice: correlation with HPLC quantitation of RPE lipofuscin and measurement of retina outer nuclear layer thickness. Invest Ophthalmol Vis Sci 2013, 54:2812-2820

59. Ng TF, Streilein JW: Light-induced migration of retinal microglia into the subretinal space. Invest Ophthalmol Vis Sci 2001, 42: $3301-3310$

60. Chen M, Forrester JV, Xu H: Dysregulation in retinal parainflammation and age-related retinal degeneration in CCL2 or CCR2 deficient mice. PLoS One 2011, 6:e22818

61. Gupta N, Brown KE, Milam AH: Activated microglia in human retinitis pigmentosa, late-onset retinal degeneration, and age-related macular degeneration. Exp Eye Res 2003, 76:463-471

62. Penfold PL, Liew SC, Madigan MC, Provis JM: Modulation of major histocompatibility complex class II expression in retinas with agerelated macular degeneration. Invest Ophthalmol Vis Sci 1997, 38: $2125-2133$

63. van der Schaft TL, Mooy CM, de Bruijn WC, de Jong PT: Early stages of age-related macular degeneration: an immunofluorescence and electron microscopy study. Br J Ophthalmol 1993, 77:657-661

64. Ma W, Zhao L, Fontainhas AM, Fariss RN, Wong WT: Microglia in the mouse retina alter the structure and function of retinal pigmented epithelial cells: a potential cellular interaction relevant to AMD. PLoS One 2009, 4:e7945

65. Raoul W, Feumi C, Keller N, Lavalette S, Houssier M, BeharCohen F, Combadiere C, Sennlaub F: Lipid-bloated subretinal microglial cells are at the origin of drusen appearance in CX3CR1deficient mice. Ophthalmic Res 2008, 40:115-119
66. Kerur N, Hirano Y, Tarallo V, Fowler BJ, Bastos-Carvalho A, Yasuma T, Yasuma R, Kim Y, Hinton DR, Kirschning CJ, Gelfand BD, Ambati J: TLR-independent and P2X7-dependent signaling mediate Alu RNA-induced NLRP3 inflammasome activation in geographic atrophy. Invest Ophthalmol Vis Sci 2013, 54: $7395-7401$

67. Hu SJ, Calippe B, Lavalette S, Roubeix C, Montassar F, Housset M, Levy O, Delarasse C, Paques M, Sahel JA, Sennlaub F, Guillonneau X: Upregulation of P2RX7 in Cx3cr1-deficient mononuclear phagocytes leads to increased interleukin-1beta secretion and photoreceptor neurodegeneration. J Neurosci 2015, 35:6987-6996

68. Olivier E, Dutot M, Regazzetti A, Leguillier T, Dargere D, Auzeil N, Laprevote O, Rat P: P2X7-pannexin-1 and amyloid beta-induced oxysterol input in human retinal cell: role in age-related macular degeneration? Biochimie 2016, 127:70-78

69. Guha S, Baltazar GC, Coffey EE, Tu LA, Lim JC, Beckel JM, Patel S, Eysteinsson T, Lu WN, O'Brien-Jenkins A, Laties AM, Mitchell $\mathrm{CH}$ : Lysosomal alkalinization, lipid oxidation, and reduced phagosome clearance triggered by activation of the $\mathrm{P} 2 \mathrm{X} 7$ receptor. FASEB J 2013, 27:4500-4509

70. Yang DL, Elner SG, Clark AJ, Hughes BA, Petty HR, Elner VM: Activation of $\mathrm{P} 2 \mathrm{X}$ receptors induces apoptosis in human retinal pigment epithelium. Invest Ophthalmol Vis Sci 2011, 52:1522-1530

71. Vessey KA, Fletcher EL: Rod and cone pathway signalling is altered in the P2X7 receptor knock out mouse. PLoS One 2012, 7:290-305

72. Fernandez-Godino R, Garland DL, Pierce EA: Isolation, culture and characterization of primary mouse RPE cells. Nat Protoc 2016, 11: $1206-1218$

73. Smart ML, Gu B, Panchal RG, Wiley J, Cromer B, Williams DA, Petrou S: P2X7 receptor cell surface expression and cytolytic pore formation are regulated by a distal C-terminal region. J Biol Chem 2003, 278:8853-8860 